\title{
Reduction in Principal Bundles: Covariant Lagrange-Poincaré Equations
}

\author{
M. Castrillón López ${ }^{1}$, T. S. Ratiu ${ }^{2}$ \\ 1 Departamento de Geometría y Topología, Universidad Complutense de Madrid, 28040 Madrid, Spain. \\ E-mail: mcastri@mat.ucm.es \\ 2 Institut de Mathématiques Bernoulli, École Polytechnique Fédérale de Lausanne, 1015 Lausanne, \\ Switzerland. E-mail: tudor.ratiu@epfl.ch
}

Received: 18 September 2001 / Accepted: 2 December 2002

Published online: 21 February 2003 - (C) Springer-Verlag 2003

\begin{abstract}
A general reduction theory for field theoretical Lagrangians on principal fiber bundles is presented. The reduced variational problem as well as the associated equations are formulated. The link between the solutions of the original problem and that of the reduced problem is discussed and an obstruction to the reconstruction of the solutions is isolated. The important case of semidirect products is discussed in detail and several concrete examples are presented.
\end{abstract}

\section{Introduction}

A standard tool in classical mechanics is the reduction method. This process, present already in the work of the founders of classical mechanics who used it to great effect in several concrete examples, uses a group of symmetries acting on the phase space of the problem in order to eliminate variables. The modern formulation of this method in the Hamiltonian context goes back to Marsden and Weinstein and is intimately related to symplectic geometry. Considerably newer is its counterpart in the Lagrangian formulation of mechanics, although its roots go back to Poincaré [24] and Hamel [11]. The simplest example of this Lagrangian reduction is the so-called Euler-Poincaré reduction, where the phase space is a Lie group $G$ which coincides with the group of symmetries of the problem (for an elementary presentation, see [15, Chapter 13]). This paradigmatic example leads to a general geometric description of Lagrangian reduction theory in [5].

The first attempt to formulate a theory of Lagrangian reduction for general variational problems on an arbitrary bundle $\pi_{M E}: E \rightarrow M$ over base manifolds $M$ of dimension not necessarily equal to 1 (which is the case of classical mechanics), is given in [4]. This paper can be considered as the equivalent of Euler-Poincaré reduction for field theories. In this case, the bundle is a principal fiber bundle $\pi_{M P}: P \rightarrow M$ with structure group $G$, which is also the group of symmetries of the field theoretical Lagrangian.

The goal of the present paper is to present reduction theory for principal fiber bundles when the group of symmetries is a subgroup $H$ of the structure group $G$. We do this 
for several reasons. First, this case represents the next natural step from the work done in [4] towards a complete theory of Lagrangian reduction. Second, the reduction by subgroups $H \subset G$ is closely related to homogeneous spaces, which is a very important case in many concrete physical and mathematical applications. This paper fulfills thus the program outlined in [4]. Finally, the study of Lagrangian reduction by stages and many examples of field theoretical Lagrangian reductions fit in this context. We present some of them at the end of the paper.

The structure of the paper is as follows. Section 2 presents a brief account of some results of the theory of principal bundles, jet manifolds, and variational calculus, needed in the sequel. Section 3 studies the geometry of the bundle $\left(J^{1} P\right) / H$, which is the configuration bundle of the reduced problem. For this purpose, a fixed connection on the bundle $P \rightarrow P / H$ is used. Section 4 formulates and proves the reduced variational principle. It turns out that this reduced variational problem is no longer free. This is coherent with the well known fact that, in general, the reduced equations are not the Euler-Lagrange equations of the reduced Lagrangian (see for example [1]). We thus obtain a new kind of equations, which can be considered as the Lagrange-Poincaré equations (see [5]) for field theories on principal bundles. Section 5 presents the reconstruction process, that is, the way to obtain the solutions of the original problem from the solutions of the reduced problem. It is shown that not every solution of the latter gives a solution to the former. Some compatibility conditions must be imposed on the reduced solutions in order to guarantee the existence of the original solutions. These conditions are formulated geometrically in terms of the flatness of the given connection. There is no equivalent to this obstruction in classical mechanics and it appears only for variational problems over manifolds whose dimension strictly exceeds 1. Section 6 links these results with those given in [4]. Section 7 presents Lagrangian semidirect product reduction for field theories. This formulation is useful when the symmetry group $H$ can be enlarged to the total group $G$ by adding new variables to the configuration bundle. This situation can be found in physics quite often; for classical mechanics it is given in [13]. Finally, Sect. 8 presents several concrete examples. The reduced equations for some of these problems can already be found in the literature, but were originally obtained ad hoc, case by case. The present theory unifies them and derives these equations from general principles.

\section{Preliminaries}

Throughout this paper, differentiability will mean $C^{\infty}$. The action of a Lie group or a Lie algebra on a manifold is denoted by concatenation of symbols or by a dot. Given the action of a Lie group $L$ on a manifold $M,\{x\}_{L}$ will denote the orbit of this action through $x \in M$, thought of as a point in the orbit space $M / L$. For the expressions in local coordinates, the Einstein summation convention of repeated indices will be used. The space of smooth sections of a given smooth locally trivial fiber bundle $E \rightarrow M$ will be denoted by $\Gamma(E)$.

If $f: M \rightarrow N$ is a smooth map between the manifolds $M$ and $N$, its derivative, or tangent map, is denoted by $T f: T M \rightarrow T N$, where $T M$ and $T N$ denote the tangent bundles of $M$ and $N$ respectively. If $\pi_{M E}: E \rightarrow M$ and $\pi_{M F}: F \rightarrow M$ are two smooth locally trivial fiber bundles over the same base $M$ the fibered product $E \times{ }_{M} F:=\left\{(u, v) \in E \times F \mid \pi_{M E}(u)=\pi_{M F}(v)\right\}$ is also a smooth locally trivial fiber bundle over $M$ whose fiber at $x \in M$ equals the product $E_{x} \times F_{x}$ of the fibers of $E$ and $F$. 


\subsection{The geometry of principal bundles}

2.1.1. Gauge transformations. Given a (right) principal $G$-bundle $\pi_{M P}: P \rightarrow M$, a gauge transformation is a diffeomorphism $\Phi: P \rightarrow P$ satisfying $\pi_{M P} \circ \Phi=\pi_{M P}$ and $\Phi(p g)=\Phi(p) g$, for all $p \in P$ and $g \in G$. The set of all gauge transformations Gau $P$ of the principal bundle $P$ is an infinite dimensional Lie group under composition. A vector field $X \in \mathfrak{X}(P)$ is said to be $G$-invariant if $\left(R_{g}\right)_{*} X=X$, for all $g \in G$, where $R_{g}: P \rightarrow P$ denotes the right free action of $G$ on $P$. The Lie algebra gau $P$ of infinitesimal gauge transformations consists of $\pi_{M P}$-vertical $G$-invariant vector fields on $P$; it is the Lie algebra of Gau $P$. If $V P$ denotes the subbundle of $T P$ of $\pi_{M P}$-vertical vectors, the quotient $(V P) / G$ turns out to be a vector bundle over $M$ and $\Gamma((V P) / G)$ is naturally identified with gau $P$.

2.1.2. Adjoint bundle. Let $\tilde{\mathfrak{g}}=(P \times \mathfrak{g}) / G \rightarrow M$ (also denoted by ad $P$ in the literature) be the adjoint bundle of $P \rightarrow M$, that is, the associated bundle defined by the adjoint action of $G$ on its Lie algebra $\mathfrak{g}$. Recall that the $G$ action on $P \times \mathfrak{g}$ is given by $(p, B) g:=\left(p g, \operatorname{Ad}_{g^{-1}} B\right)$. The elements of $\tilde{\mathfrak{g}}$ are denoted by $\{p, B\}_{G}$, for $p \in P$, $B \in \mathfrak{g}$. The Lie bracket $[\cdot, \cdot]$ on $\mathfrak{g}$ endows the fibers of $\tilde{\mathfrak{g}}$ with the structure of a Lie algebra defined by

$$
\left[\{p, B\}_{G},\left\{p, B^{\prime}\right\}_{G}\right]:=\left\{p,\left[B, B^{\prime}\right]\right\}_{G}, \quad p \in P, \quad B, B^{\prime} \in \mathfrak{g},
$$

which depends smoothly on the base point. Thus $\tilde{\mathfrak{g}}$ is a bundle of Lie algebras.

Given an element $B$ of the Lie algebra $\mathfrak{g}$ of $G$, the infinitesimal generator of the $G$-action on $P$ is denoted by $B^{*} \in \mathfrak{X}(P)$, that is, $B_{p}^{*}:=d(p \exp t B) /\left.d t\right|_{t=0}$, for any $p \in P$. The diffeomorphism $P \times \mathfrak{g} \rightarrow V P,(p, B) \mapsto B_{p}^{*}$, induces an isomorphism between the Lie algebra $\Gamma(\tilde{\mathfrak{g}})$ of sections of the adjoint bundle $\tilde{\mathfrak{g}} \rightarrow M$ and the Lie algebra of infinitesimal gauge transformations gau $P$, that is, we have $\Gamma(\tilde{\mathfrak{g}}) \cong \Gamma((V P) / G)=$ gau $P$. In what follows, we shall not distinguish between these two Lie algebras.

2.1.3. Connections on $P$. A connection on $P$ is, by definition, a smooth $G$-invariant vector subbundle $\mathcal{H}$ of $T P$ such that $\mathcal{H} \oplus V P=T P$. A connection is equivalently characterized by its connection 1 -form $\omega^{\mathcal{H}}$, which is an equivariant $\mathfrak{g}$-valued 1 -form defining the vertical part of a vector field by means of the identification $V P \cong P \times \mathfrak{g}$. That is, $\omega^{\mathcal{H}}$ satisfies $\omega^{\mathcal{H}}(p g)=\operatorname{Ad}_{g^{-1}} \circ \omega^{\mathcal{H}}(p)$ for all $p \in P, g \in G$ and $\omega^{\mathcal{H}}(p)\left(B_{p}^{*}\right)=B$, for all $B \in \mathfrak{g}$ and $p \in P$. The connection $\mathcal{H}$ defines the horizontal lift operator $\cdot \mathcal{H}: T_{x} M \rightarrow T_{p} P$, $Y \mapsto Y^{\mathcal{H}}, x \in M, p \in \pi_{M P}^{-1}(x)$ by the requirement that $Y^{\mathcal{H}}$ is the only vector of $\mathcal{H}_{p}$ that projects to $Y$, that is, $T_{p} \pi_{M P}\left(Y^{\mathcal{H}}\right)=Y$.

The space of connections of a principal bundle is an infinite dimensional affine space modeled over the vector space $\Omega^{1}(M, \tilde{\mathfrak{g}})$ of $\tilde{\mathfrak{g}}$-valued 1 -forms on $M$.

2.2. The geometry of $P / H$. Let $\pi_{M P}: P \rightarrow M$ be a principal fiber bundle with structure group $G$ and let $H$ be a Lie subgroup of $G$. The quotient $\Sigma:=P / H$ of $P$ under the right action of $H$ is a manifold and the projection $\pi_{\Sigma P}: P \rightarrow P / H$ is a principal $H$-bundle. The projection $\pi_{M \Sigma}: P / H \rightarrow M,\{p\}_{H} \mapsto \pi_{M P}(p)$, has the structure of fiber bundle over $M$ with typical fiber $G / H$; the elements of $G / H$ are the equivalence classes $\{g\}_{H}=g H$. We have hence the following. 
Proposition 1 ([14, I. Prop. 5.5]). The bundle $P \times{ }_{G}(G / H)=(P \times(G / H)) / G$ associated to $P$ with respect to the natural left action of $G$ on $G / H$, can be identified with $P / H$. The identification is given by

$$
(P \times(G / H)) / G \rightarrow P / H, \quad\left\{p,\{g\}_{H}\right\}_{G} \mapsto\{p g\}_{H}
$$

Throughout the paper, we will be concerned with the space of sections of the fiber bundle $P / H \rightarrow M$. These global sections enjoy an interesting property: they define structure group reductions of the bundle $P \rightarrow M$. More precisely, we say that the $G$-principal bundle $P \rightarrow M$ is $H$-reducible if there exists a subbundle $P^{H}$ of $P$ that is simultaneously a principal bundle with structure group $H$ and base $M$.

Proposition 2 ([14, I. Prop. 5.6]). Let $H$ be a Lie subgroup of a Lie group $G$ and let $P \rightarrow M$ be a G-principal bundle. Then there is a bijective correspondence between $H$-reductions $P^{H}$ of $P \rightarrow M$ and global sections of the bundle $\pi_{M \Sigma}: P / H \rightarrow M$. The $H$-reduction associated to a section $\varsigma: M \rightarrow P / H$ is $P^{\varsigma}=\pi_{\Sigma P}^{-1}(\varsigma(M))$, that is, this bundle coincides with the pull-back bundle $\varsigma^{*} P$ of the $H$-principal bundle $P \rightarrow P / H$ by 5 .

The identification between $P^{\varsigma}=\pi_{\Sigma P}^{-1}(\varsigma(M))$ and $\varsigma^{*} P$ is given by $p \in \pi_{\Sigma P}^{-1}(\varsigma(M)) \leftrightarrow$ $\left(p, \pi_{M P}(p)\right) \in \varsigma^{*} P$.

2.3. The space of jets. For the material in this subsection we refer the reader, for example, to [27]. We shall recall here only the notations and shall fix the conventions in force throughout the paper. Let $\pi_{M E}: E \rightarrow M$ be a smooth locally trivial fiber bundle. Two local sections $s, s^{\prime}$ of $\pi$ are said to have the same 1-jet at $x \in M$ if $s(x)=s^{\prime}(x)$ and $T_{x} s=T_{x} s^{\prime}$. The equivalence classes are denoted by $j_{x}^{1} s$ and the set of all equivalence classes at $x \in M$ is denoted by $J_{x}^{1} E$. The set $J^{1} E:=\bigcup_{x \in M} J_{x}^{1} E$ can be endowed with the structure of a smooth affine fiber bundle over $E$ with projection map $\pi_{10}: J^{1} E \rightarrow E$ defined by $\pi_{10}\left(j_{x}^{1} s\right)=s(x)$. If $\operatorname{dim} M=n$ and $\operatorname{dim} E=n+m$, then $\operatorname{dim} J^{1} E=n+m+n m$. Let $\left(x^{i}, y^{\alpha}\right), 1 \leq i \leq n, 1 \leq \alpha \leq m$, be a fibered coordinate system on $U \subset E$. The induced natural chart $\left(x^{i}, y^{\alpha}, y_{i}^{\alpha}\right)$ on $\pi_{10}^{-1}(U) \subset J^{1} E$ is defined by

$$
y_{i}^{\alpha}\left(j_{x}^{1} s\right):=\left.\frac{\partial\left(y^{\alpha}(s)\right)}{\partial x^{i}}\right|_{x} .
$$

Remark 1. There is a different but equivalent description of the 1 -jet space $J^{1} E$. Given a class $j_{x}^{1} s \in J^{1} E$ the tangent mapping $T_{x} s: T_{x} M \rightarrow T_{s(x)} E$ is well defined since $T_{x} s$ depends only on the first derivative of $s$ at $x$. Similarly, given a linear map $\lambda: T_{x} M \rightarrow$ $T_{p} E$ such that $T_{p} \pi_{M E} \circ \lambda=\mathrm{Id}$, there is a unique $j_{x}^{1} s \in J^{1} E$ such that $T_{x} s=\lambda$. Therefore, we can realize $J^{1} E$ as the space of all linear maps $\lambda: T_{x} M \rightarrow T_{p} E, x \in M$, $p \in \pi_{M E}^{-1}(x)$, verifying $T_{p} \pi_{M E} \circ \lambda=$ Id. Actually, we can go further and we can identify $j_{x}^{1} s$ with the range $T_{x} s\left(T_{x} M\right) \subset T_{s(x)} E$. In this way, $J^{1} E$ can be thought of as the set of subspaces $\mathcal{H}_{p} \subset T_{p} E, p \in E$, such that $\mathcal{H}_{p} \oplus V_{p} E=T_{p} E$, where $V_{p} E$ is the subspace of vertical vectors at $p \in E$. This interpretation of 1-jets will be convenient in the sequel. 
Given a smooth morphism of fiber bundles $\Phi: E \rightarrow E$, covering the diffeomorphism $\varphi: M \rightarrow M$, also called a fibered mapping over $\varphi$, the 1-jet extension $\Phi^{(1)}$ : $J^{1} E \rightarrow J^{1} E$ of $\Phi$ is defined by $\Phi^{(1)}\left(j_{x}^{1}(s)\right):=j_{\varphi(x)}^{1}\left(\Phi \circ s \circ \varphi^{-1}\right)([27$, Ch. 4]). This map obviously satisfies $\Phi \circ \pi_{10}=\pi_{10} \circ \Phi^{(1)}$. In particular, if $\Phi$ is vertical (that is, $\pi_{M E} \circ \Phi=\pi_{M E}$, or, equivalently, $\varphi=$ identity on $M$ ), the definition of $\Phi^{(1)}$ is simply $\Phi^{(1)}\left(j_{x}^{1} s\right)=j_{x}^{1}(\Phi \circ s), j_{x}^{1} s \in J^{1} E$.

Let $\mathfrak{X}^{v}(E):=\left\{X \in \mathfrak{X}(E) \mid T \pi_{M E} \circ X=0\right\}$ denote the Lie algebra of vertical vector fields on $E$. Thus, given a $\pi_{M E}$-vertical vector field $X \in \mathfrak{X}^{v}(E)$ with flow $\Phi_{t}$, define $X^{(1)} \in \mathfrak{X}\left(J^{1} E\right)$ by the requirement that its flow be $\Phi_{t}^{(1)}$. The mapping $\mathfrak{X}^{v}(E) \rightarrow \mathfrak{X}\left(J^{1} E\right), X \mapsto X^{(1)}$, is a homomorphism of Lie algebras called the natural lift to $J^{1} E$ or shortly, the 1-jet lift of vector fields.

Given a section $s: M \rightarrow E$ of $\pi_{M E}: E \rightarrow M$, denote by $j^{1} s: M \rightarrow J^{1} E$ the mapping sending each $x \in M$ to $j_{x}^{1} s$.

2.4. Calculus of variations. Given a fiber bundle $\pi_{M E}: E \rightarrow M$, a first order Lagrangian density is a fibered morphism $\mathcal{L}: J^{1} E \rightarrow \bigwedge^{n} T^{*} M$ over the identity mapping on $M$. Assuming that $M$ is oriented by a volume form $v$ (i.e., $M$ is orientable), we can write $\mathcal{L}\left(j_{x}^{1} s\right)=L\left(j_{x}^{1} s\right) v_{x}, j_{x}^{1} s \in J^{1} E$, where $L: J^{1} E \rightarrow \mathbb{R}$ is called the Lagrangian associated to $\mathcal{L}$.

Denote by $\Gamma_{c}(E)$ the set of compactly supported sections of the fiber bundle $\pi_{M E}$ : $E \rightarrow M$. The action defined by a Lagrangian density is the mapping

$$
\mathcal{S}: \Gamma_{c}(E) \rightarrow \mathbb{R},
$$

sending a (local) section $s$ of $\pi_{M E}: E \rightarrow M$ with compact support in the open set $U \subset M$ to the value $\int_{U} \mathcal{L} \circ j^{1} s=\int_{U}\left(L \circ j^{1} s\right) v$. Given such a compactly supported section $s: U \rightarrow E$, a vertical variation of $s$ is a one parameter family $s_{\varepsilon}$ of sections of $\pi_{M E}: E \rightarrow M$, each $s_{\varepsilon}$ defined on $U$ and such that $s_{0}=s$. The derivative

$$
\delta s(x):=\left.\frac{d s_{\varepsilon}(x)}{d \varepsilon}\right|_{\varepsilon=0},
$$

is called the infinitesimal variation of $s_{\varepsilon} ; \delta s$ is a vector field covering the section $s$, vertical with respect to $\pi_{M E}$, that is, $\delta s: M \rightarrow T E$ satisfies $\delta s(x) \in T_{s(x)} E$ and $T \pi_{M E} \circ \delta s=0$. Since the section $s$ has compact support in the open set $U$, we will always consider variations $s_{\varepsilon}$ such that $\delta s=0$ on the boundary $\partial U$. If $M$ is compact, we can consider global sections and the requirement $\delta s=0$ on $\partial U$ is no longer necessary. We say that $s: U \rightarrow E$ is a critical section of the variational problem defined by $\mathcal{L}$ if

$$
\delta \int_{U} \mathcal{L} \circ j^{1} s:=\left.\frac{d}{d \varepsilon}\right|_{\varepsilon=0} \int_{U} \mathcal{L} \circ j^{1} s_{\varepsilon}=\left.\frac{d}{d \varepsilon}\right|_{\varepsilon=0} \int_{U}\left(L \circ j^{1} s_{\varepsilon}\right) v=0,
$$

for every variation $s_{\varepsilon}$. If $X \in \mathfrak{X}^{v}(E)$ is a vertical vector field such that

$$
\left.\frac{d s(x)}{d \varepsilon}\right|_{\varepsilon=0}=X_{x}, \quad \text { for all } x \in U,
$$

we have (see for example [10])

$$
\left.\frac{d}{d \varepsilon}\right|_{\varepsilon=0} \int_{U}\left(L \circ j^{1} s_{\varepsilon}\right) v=\int_{U} \frac{\delta L}{\delta s}\left(X^{(1)}\right) v,
$$


where $\delta L / \delta s$ denotes the differential along $j^{1} s$, that is,

$$
\frac{\delta L}{\delta s}(Z)=\left(i_{Z} \mathrm{~d} L\right) \circ j^{1} s,
$$

for vector fields $Z \in \mathfrak{X}\left(J^{1} E\right)$ vertical with respect to the projection $\pi_{M E} \circ \pi_{10}$. By $i_{Z}$ we denote the interior product (contraction on the first index) with $Z$. Hence, a section is critical if and only if

$$
\int_{U} \frac{\delta L}{\delta s}\left(X^{(1)}\right) v=0
$$

for all $X \in \mathfrak{X}^{v}(E)$ with compact support.

Remark 2. As we have already noted, for first order variational calculus, the derivative $d \int_{U}\left(L \circ j^{1} s_{\varepsilon}\right) v /\left.d \varepsilon\right|_{\varepsilon=0}$ depends only on the infinitesimal variation $\delta s$ and not on the variation of $\mathrm{s}$ itself; that is, two variations $s_{\varepsilon}$ and $s_{\varepsilon}^{\prime}$ with $\delta s=\delta s^{\prime}$ will give the same value of the derivative. We will use this property very often, especially in the study of the variations of the reduced variational problem.

Given a section $s$, the vertical differential $\delta L / \delta s$ can be seen as a differential operator on vectors $X \in \mathfrak{X}^{v}(E)$. The adjoint differential operator $\mathcal{E} \mathcal{L}(L)$ is a section of $V^{*} E$ (the dual to the vertical subbundle) and is called the Euler-Lagrange operator defined by $L$ along the section $s$ (see for example [10]). The local expression of this operator is

$$
\mathcal{E} \mathcal{L}(L)=\left\{\frac{\partial L}{\partial y^{\alpha}} \circ j^{1} s+\frac{\partial}{\partial x^{i}}\left(\frac{\partial L}{\partial y_{i}^{\alpha}} \circ j^{1} s\right)\right\} \mathrm{d} y^{\alpha} .
$$

The equation $\mathcal{E} \mathcal{L}(L)=0$ is known as the Euler-Lagrange equation for $s$ and is equivalent to the fact that $s$ is an extremal.

Remark 3. In the setup of the calculus of variations presented above we have worked with compactly supported sections, but in many situations the classical solutions are not compactly supported. What is really important is the compactness of the support of the variations, the key property with which the variational equations can still be defined. For the sake of simplicity and in order to avoid the introduction of further notations, we have opted to work in this paper with sections with compact support and, even more, we will often assume that the manifold $M$ is itself compact, with or without boundary, and that the sections $s$ under consideration are global sections. These assumptions, while considerably simplifying the exposition, do not represent real restrictions to the theory and can be omitted, if desired, with minor notational modifications. Also, every time this appears, the result is really local, so this issue of compactness is not really relevant.

\section{The Geometry of $\left(J^{1} P\right) / H$}

3.1. The identification $\left(J^{1} P\right) / H=J^{1}(P / H) \times{ }_{P / H}\left(T^{*} M \otimes \tilde{\mathfrak{h}}\right)$. Given a principal $G$-bundle, the right action $R$ of $G$ on $P$ gives rise to a right action on $J^{1} P$ in a natural way, namely

$$
j_{x}^{1} s \cdot g:=j_{x}^{1}\left(R_{g} \circ s\right), \quad j_{x}^{1} s \in J^{1} P, \quad g \in G .
$$


We are concerned with the structure of the quotient set $\left(J^{1} P\right) / H$. An element $\left\{j_{x}^{1} s\right\}_{H} \in$ $\left(J^{1} P\right) / H$ can be seen as a $H$-invariant distribution of vector subspaces complementary to the vertical bundle $V P$ (see Sect. 2.3 above). Indeed, given $j_{x}^{1} s \in J^{1} P$, the class $\left\{j_{x}^{1} s\right\}_{H}$ represents a distribution along the $H$-orbit of $p=s(x)$, which, at the point $p h$, $h \in H$, is given by $\left(T_{p} R_{h} \circ T_{x} s\right)\left(T_{x} M\right) \subset T_{p h} P$.

The space $\left(J^{1} P\right) / H$ is a differentiable manifold and the canonical projection $J^{1} P \rightarrow$ $\left(J^{1} P\right) / H$ endows it with the structure of a $H$-principal bundle. The geometry of the space $\left(J^{1} P\right) / H$ is better understood with the aid of a connection $\mathcal{H}$ on the $H$-principal bundle $\pi_{\Sigma P}: P \rightarrow P / H=: \Sigma$ and its associated connection one-form $\omega^{\mathcal{H}}$. Let $\tilde{\mathfrak{h}}$ be the adjoint bundle of $P \rightarrow P / H$, that is, $\tilde{\mathfrak{h}}=(P \times \mathfrak{h}) / H$. The one-form $\omega^{\mathcal{H}}$ defines a homomorphism $\tilde{\omega}^{\mathcal{H}}: T P \rightarrow \tilde{\mathfrak{h}}$ of vector bundles over $\Sigma=P / H$ given by

$$
\tilde{\omega}^{\mathcal{H}}(X):=\left\{p, \omega^{\mathcal{H}}(X)\right\}_{H}, \quad \text { for all } \quad X \in T_{p} P, p \in P .
$$

It is straightforward to check that $\tilde{\omega}^{\mathcal{H}} \circ T R_{h}=\tilde{\omega}^{\mathcal{H}}$, for all $h \in H$. We know that sections of $\tilde{\mathfrak{h}}$ correspond to $H$-invariant vertical vector fields (see Sect. 2.1.2). Similarly, an element $\{p, B\}_{H} \in \tilde{\mathfrak{h}}$ will correspond to a $H$-invariant $\pi_{\Sigma P}$-vertical vector field along the fiber $\pi_{\Sigma P}^{-1}\left(\{p\}_{H}\right)$. Thus, $\tilde{\omega}^{\mathcal{H}}$ when applied to a vector $X \in T_{p} P$, gives the $H$-invariant $\pi_{\Sigma} P$-vertical vector field along the $H$-orbit $\operatorname{orb}^{H}(p)$ through $p$ whose value at $p$ equals the vertical part of $X$.

We now define the fibered morphism over $P / H$

$$
\phi_{\mathcal{H}}:\left(J^{1} P\right) / H \rightarrow J^{1}(P / H) \times_{P / H}\left(\pi_{M \Sigma}^{*} T^{*} M \otimes_{P / H} \tilde{\mathfrak{h}}\right),
$$

given by

$$
\phi_{\mathcal{H}}\left(\left\{j_{x}^{1} s\right\}_{H}\right):=\left(j_{x}^{1}\{s\}_{H}, \tilde{\omega}^{\mathcal{H}} \circ T_{x} s\right),
$$

where $x \in M$ and $\{s\}_{H} \in \Gamma(P / H)$ is the local section of $P / H \rightarrow M$ induced by the local section $s$ of $P \rightarrow M$.

Proposition 3. The fibered morphism over $\Sigma=P / H$,

$$
\phi_{\mathcal{H}}:\left(J^{1} P\right) / H \rightarrow J^{1}(P / H) \times_{P / H}\left(\pi_{M \Sigma}^{*} T^{*} M \otimes_{P / H} \tilde{\mathfrak{h}}\right),
$$

is a diffeomorphism and hence a fiber bundle isomorphism.

Proof. First we check that $\phi_{\mathcal{H}}$ is well defined. If $s^{\prime}=R_{h} \circ s, h \in H$, is another representative of the class $\left\{j_{x}^{1} s\right\}_{H}$, then it follows that $\left\{s^{\prime}\right\}_{H}=\{s\}_{H}$ and $\omega^{\mathcal{H}} \circ T s^{\prime}=$ $\omega^{\mathcal{H}} \circ T R_{h} \circ T s=\omega^{\mathcal{H}} \circ T s$. Clearly, $\phi_{\mathcal{H}}$ is differentiable. For the inverse, we define the morphism

$$
\psi_{\mathcal{H}}: J^{1}(P / H) \times_{P / H}\left(\pi_{M \Sigma}^{*} T^{*} M \otimes_{P / H} \tilde{\mathfrak{h}}\right) \rightarrow\left(J^{1} P\right) / H,
$$

by

$$
\psi_{\mathcal{H}}\left(j_{x}^{1}\{s\}_{H}, \xi\right)=\left\{j_{x}^{1} \hat{s}\right\}_{H}
$$

where $j_{x}^{1} \hat{s} \in J^{1} P$ is determined by its point value $\hat{s}(x)=p \in \pi_{\Sigma P}^{-1}\left(\{s(x)\}_{H}\right)$ and by the value of its derivative $T \hat{s}: T_{x} M \rightarrow T_{p} P, T \hat{s}(X)=\left(T\{s\}_{H}(X)\right)^{\mathcal{H}}+\xi(X)_{p}$; here . H $: T_{\{s(x)\}_{H}} \Sigma \rightarrow T_{p} P$ denotes the horizontal lift operator and $\xi(X) \in \tilde{\mathfrak{h}}$ is interpreted 
as a $H$-invariant vector field along $\pi_{\Sigma P}^{-1}\left(\{s(x)\}_{H}\right)$ evaluated at the point $p \in P$. The definition does not depend on the choice of the point $p \in \pi_{\Sigma P}^{-1}\left(\{s(x)\}_{H}\right)$. The differentiability of $\psi_{\mathcal{H}}$ as well as the identities $\phi_{\mathcal{H}} \circ \psi_{\mathcal{H}}=\operatorname{Id}$ and $\psi_{\mathcal{H}} \circ \phi_{\mathcal{H}}=\operatorname{Id}$ are easy to check.

Notation. In the sequel, we will make use of the identification defined by $\phi_{\mathcal{H}}$,

$$
\left(J^{1} P\right) / H \cong J^{1}(P / H) \times{ }_{P / H}\left(T^{*} M \otimes \tilde{\mathfrak{h}}\right),
$$

without an explicit reference to the connection $\mathcal{H}$. Moreover, for the sake of simplicity, we directly write $T^{*} M \otimes \tilde{\mathfrak{h}}$ instead of the bundle $\pi_{M \Sigma}^{*} T^{*} M \otimes_{P / H} \tilde{\mathfrak{h}}$, where the pull-back notation is omitted as it is clear that all the vector bundles and the tensor product are understood over $P / H$.

Remark 4. It is important to note that the identification given in Proposition 3 is not canonical and depends on the choice of the connection $\mathcal{H}$. Roughly speaking, the identification (3.2) gives a splitting of every element $\left\{j_{x}^{1} s\right\}_{H} \in\left(J^{1} P\right) / H$ in the horizontal and vertical part $\left(j_{x}^{1}\{s\}_{H}\right.$ and $\tilde{\omega}^{\mathcal{H}} \circ T s$, respectively) relative to the connection $\mathcal{H}$. Though the horizontal part is independent of $\mathcal{H}$, the vertical part does depend on $\mathcal{H}$, as it gives the vertical component of $T s$ with respect to $\mathcal{H}$.

3.2. The bundle $T^{*} M \otimes \tilde{\mathfrak{h}} \rightarrow M . \quad$ We study now the composite bundle

$$
T^{*} M \otimes \tilde{\mathfrak{h}} \stackrel{\pi_{\Sigma \mathfrak{h}}}{\longrightarrow} P / H=\Sigma \stackrel{\pi_{M \Sigma}}{\longrightarrow} M .
$$

We will see later that the critical sections of the reduced variational problem are in fact sections of this bundle.

A section $\sigma: M \rightarrow T^{*} M \otimes \tilde{\mathfrak{h}}$ of this composite bundle induces two geometrical objects over $M$ :

- First, we obtain a section $\varsigma: M \rightarrow P / H, \varsigma(x):=\pi_{\Sigma \mathfrak{h}}(\sigma(x))$, and therefore, by virtue of Proposition 2, a submanifold $P^{\varsigma} \subset P$ which is a $H$-reduction of the bundle $P \rightarrow M$. This bundle is the pull-back bundle $\varsigma^{*} P$ of the bundle $P \rightarrow P / H$. Given the fixed connection $\mathcal{H}$ on $P \rightarrow P / H$ we consider its restriction to $P^{\varsigma}$, which is also a connection in the principal $H$-bundle $P^{\varsigma} \rightarrow M$.

- Second, the section $\sigma$ can be interpreted as a $\varsigma^{*} \tilde{\mathfrak{h}}$-valued 1-form on $M$. Since the adjoint bundle of $P^{\varsigma} \rightarrow M$ is $\varsigma^{*} \tilde{\mathfrak{h}}$, we can subtract this form from $\omega^{\mathcal{H}}$ and we thus obtain a new connection $\mathcal{H}^{\sigma}$ on $P^{\varsigma}$.

Remark 5. In fact, what we have described above is the configuration space used for the geometrical description of the process known as spontaneous symmetry breaking (see [9 Sect. 3.8 26, 21] for a complete description of this construction). Roughly speaking, this quantum phenomenon is modeled as follows. We have an original $G$-principal bundle which models a particular physical system. We have a subgroup $H \subset G$. The symmetry is said to be "broken" when we choose a $H$-reduction $P^{\varsigma}$ of $P$ induced by a section $\varsigma: M \rightarrow P / H$. Frequently in gauge theories, the new configuration bundle $P^{S}$ is endowed with a potential, that is, a connection $\mathcal{H}^{\sigma}$ on it. Thus, it is precisely the sections of the composite fiber bundle $T^{*} M \otimes \tilde{\mathfrak{h}} \rightarrow M$ who describe all possible couplings of $\left(H\right.$-reductions $\left.P^{\varsigma}\right)+\left(\right.$ connections $\mathcal{H}^{\sigma}$ on $\left.P^{\varsigma}\right)$. This bundle turns out to be the ideal place to formulate variational models of this physical process as its configuration bundle. 


\section{The Reduction of the Variational Principle}

4.1. Invariant Lagrangians. We now assume that the Lagrangian $L: J^{1} P \rightarrow \mathbb{R}$ is invariant under the action of a subgroup $H \subset G$ on $J^{1} P$. Then $L$ projects to the quotient and we obtain a function

$$
l:\left(J^{1} P\right) / H \rightarrow \mathbb{R}
$$

called the reduced Lagrangian. The variational problem defined by $L$ gives a new variational problem (with constraints, as we will see below) on the quotient $\left(J^{1} P\right) / H$. The use of the identification $\left(J^{1} P\right) / H=J^{1}(P / H) \times_{P / H}\left(T^{*} M \otimes \tilde{\mathfrak{h}}\right)$, for a fixed connection $\mathcal{H}$ on $P \rightarrow P / H$, will be needed for its description; recall that we denote, for simplicity, $T^{*} M \otimes \tilde{\mathfrak{h}}:=\pi_{M \Sigma}^{*} T^{*} M \otimes_{P / H} \tilde{\mathfrak{h}}$.

We see now that the critical sections of the new problem are the sections of the bundle $T^{*} M \otimes \tilde{\mathfrak{h}} \rightarrow M$. Given a section $s$ of $\pi_{M P}: P \rightarrow M$, we define the section $\sigma: M \rightarrow T^{*} M \otimes \tilde{\mathfrak{h}}$ by

$$
\sigma(x):=\tilde{\omega}^{\mathcal{H}} \circ T_{x} s, \quad x \in M,
$$

which is called the reduced section. The section $s$ also defines a section $\varsigma=\{s\}_{H}$ of the bundle $P / H \rightarrow M$, which can also be obtained by projecting $\sigma$ to $P / H$ via $\pi_{\Sigma \mathfrak{h}}: T^{*} M \otimes \tilde{\mathfrak{h}} \rightarrow P / H$, that is

$$
\varsigma=\{s\}_{H}=\pi_{\Sigma \mathfrak{h}} \circ \sigma .
$$

If we consider jet bundles, the section $s$ naturally induces the section $j^{1} s$ of $J^{1} P \rightarrow M$, which defines, by virtue of the identification (3.2), the section

$$
\left(j^{1} \varsigma, \sigma\right): M \rightarrow J^{1}(P / H) \times_{P / H}\left(T^{*} M \otimes \tilde{\mathfrak{h}}\right) .
$$

The two components of a section of this kind are not independent as $j^{1}\{s\}_{H}=j^{1} \varsigma$ can be obtained from $\sigma$. In fact, the section $\sigma$ of the bundle $T^{*} M \otimes \tilde{\mathfrak{h}} \rightarrow M$ completely determines the section $\left(j^{1} \zeta, \sigma\right): M \rightarrow J^{1}(P / H) \times P / H\left(T^{*} M \otimes \tilde{\mathfrak{h}}\right)$. The space of these sections is where the action functional of the reduced Lagrangian $l$ is defined, namely

$$
\left(j^{1} \varsigma, \sigma\right) \mapsto \int_{M} l \circ\left(j^{1} \varsigma, \sigma\right) v .
$$

For these reasons, it is clear that the study of the reduced problem takes place on the space of sections of the composite bundle $T^{*} M \otimes \tilde{\mathfrak{h}} \rightarrow M$. This space is the configuration bundle of the reduced problem.

Let $s$ be a section of $\pi_{M P}$ and $s_{\varepsilon}$ be an arbitrary variation of $s$. The infinitesimal variation $\delta s$ of $s_{\varepsilon}$ determines a variation

$$
\delta \sigma(x)=\left.\frac{d}{d \varepsilon}\right|_{\varepsilon=0} \sigma_{\varepsilon}(x)=\left.\frac{d}{d \varepsilon}\right|_{\varepsilon=0}\left(\tilde{\omega}^{\mathcal{H}} \circ T_{x} s_{\varepsilon}\right), \quad x \in M,
$$

of the projected section $\sigma$. The variation of the action defined by $L$ gives

$$
\left.\frac{d}{d \varepsilon}\right|_{\varepsilon=0} \int_{M} L\left(j^{1} s_{\varepsilon}\right) v=\left.\frac{d}{d \varepsilon}\right|_{\varepsilon=0} \int_{M} l\left(j^{1} \varsigma_{\varepsilon}, \sigma_{\varepsilon}\right) v .
$$


Therefore, a section $\sigma$ of $\pi_{M \Sigma}^{*} T^{*} M \otimes_{P / H} \tilde{\mathfrak{h}} \equiv T^{*} M \otimes \tilde{\mathfrak{h}} \rightarrow M$ will be critical if and only if

$$
\int_{M} i_{\left(\delta \zeta^{(1)}, \delta \sigma\right)} \mathrm{d} l v=0,
$$

for any variation $\delta \sigma$ of $\sigma$ of the type described in formula (4.1) and with $\delta \zeta=$ $T \pi_{\Sigma} \delta \delta$ ). That is, the variational problem defined by $L$ becomes a variational problem on $T^{*} M \otimes \tilde{\mathfrak{h}}$ with constraints on the set of admissible variations (see also Remark 8 below).

Remark 6. The phase space of this new variational problem is a fiber product $J^{1}(P / H) \times_{P / H}\left(T^{*} M \otimes \tilde{\mathfrak{h}}\right)$ of a jet space and a vector bundle. This is not the usual case in the calculus of variations, where the phase space is always the jet bundle of the configuration bundle. This new category of phase spaces has been already observed for the case of classical mechanics in [5]. We believe that this category represents a suitable framework for a future theory of reduction by stages in Field Theory, which would be of great interest.

For the reduction of the variational principle, we need to characterize the structure of the admissible variations $\delta \sigma$. For this purpose, we make use again of the fixed connection $\mathcal{H}$. Using this connection, we will be able to distinguish vertical and horizontal variations of $\delta s$. We will give an explicit formula of $\delta \sigma$ for those cases. Finally, as an arbitrary variation $\delta s$ can be always written as the sum of a vertical and a horizontal variation $\delta s=\delta s^{v}+\delta s^{h}$, the general expression of $\delta \sigma$ will be simply the sum of $\delta \sigma^{v}+\delta \sigma^{h}$.

4.2. Vertical variations. Although an infinitesimal variation $\delta s$ of an arbitrary variation $s: M \rightarrow P$ is by definition vertical with respect to $\pi_{M P}$, it need not be relative to $\pi_{\Sigma P}$. In fact, an infinitesimal variation $\delta s$ is vertical with respect to $\pi_{\Sigma P}$ if and only if there exists a mapping $B: M \rightarrow \mathfrak{h}$ such that $\delta s(x)=B(x)_{s(x)}^{*}$ (see Sect. 2.1.2 above). That is, $\delta s$ can be seen as the infinitesimal generator of the variation $s_{\varepsilon}(x)=s(x) \exp (\varepsilon B(x))$. In this section we are going to study variations of this type.

If $\varsigma: M \rightarrow P / H$ denotes the class $\{s\}_{H}$, we see that the variation $\varsigma_{\varepsilon}$ induced by the variation $s_{\varepsilon}$ described above, does not depend on $\varepsilon$, that is $\varsigma_{\varepsilon}=\varsigma$, for all $\varepsilon$. Using the identification (3.2) with respect to the fixed connection $\mathcal{H}$, i.e. $\left(J^{1} P\right) / H \cong$ $J^{1}(P / H) \times_{P / H}\left(T^{*} M \otimes \tilde{\mathfrak{h}}\right)$, we conclude that $\left\{j^{1} s_{\varepsilon}\right\}_{H}=\left(j^{1} \varsigma_{\varepsilon}, \sigma_{\varepsilon}\right)=\left(j^{1} \varsigma, \sigma_{\varepsilon}\right)$. Hence we can say that the infinitesimal variation along $\left(j^{1} \zeta, \sigma\right)$ is the zero vector field for the jet component $J^{1}(P / H)$ and a vector field on the vector bundle $T^{*} M \otimes \tilde{\mathfrak{h}}$ along $\sigma$ for the second component. As every section $\sigma_{\varepsilon}$ of the composite bundle $T^{*} M \otimes \tilde{\mathfrak{h}}$ projects to the same section $\varsigma$ of $P / H \rightarrow M$, the variation $\delta \sigma=\left.(d / d \varepsilon)\right|_{\varepsilon=0} \sigma_{\varepsilon}$ is vertical with respect to the projection $T^{*} M \otimes \tilde{\mathfrak{h}} \rightarrow P / H$. As this last bundle is a vector bundle, we can thus interpret $\delta \sigma$ as a section of the composite bundle $T^{*} M \otimes \tilde{\mathfrak{h}} \rightarrow M$, which projects to the same section $\varsigma: M \rightarrow P / H$ as $\sigma$, that is,

$$
\delta \sigma \in \Gamma\left(T^{*} M \otimes \tilde{\mathfrak{h}}\right) .
$$

With this idea, the structure of the variation $\delta \sigma$ can be described as follows. 
Proposition 4. Given a vertical variation $\delta s$ and the mapping $B: M \rightarrow \mathfrak{h}$ such that $\delta s(x)=B(x)_{s(x)}^{*}$, let $\eta$ be the section of the bundle $\tilde{\mathfrak{h}} \rightarrow M$ defined by

$$
\eta(x)=\{s(x), B(x)\}_{H}, \quad x \in M .
$$

The section $\eta$ can be interpreted as a section of the vector bundle $\varsigma^{*} \tilde{\mathfrak{h}} \rightarrow M$. Then we have

$$
\delta \sigma=\nabla^{\mathcal{H}} \eta-[\sigma, \eta],
$$

where $\nabla^{\mathcal{H}}$ is the linear connection induced by $\mathcal{H}$ on $\tilde{\mathfrak{h}}$ (and on $\varsigma^{*} \tilde{\mathfrak{h}}$ ).

Proof. As the formula we want to prove is local and every principal bundle is locally trivial, it is enough to consider the special case $P=P / H \times H$. In this case the section $s$ can be written as $s(x)=(\varsigma(x), h(x))$, for a certain mapping $h: M \rightarrow H$. We have $T s=(T \varsigma, T h)$. We now take the variation $s_{\varepsilon}(x)=s(x) \exp (\varepsilon B(x))=$ $(\varsigma(x), h(x) \exp (\varepsilon B(x))$. The chain rule yields

$$
T s_{\varepsilon}=(T \varsigma, T(h \exp (\varepsilon B)))=\left(T \varsigma, T L_{h} \circ T \exp (\varepsilon B)+T R_{\exp (\varepsilon B)} \circ T h\right),
$$

where $L$ and $R$ stand for the left and right translation on the group $H$ respectively. On the other hand, on a trivial bundle, the composition of $T s_{\varepsilon}$ with the connection form has the expression

$$
\omega^{\mathcal{H}} \circ T s_{\varepsilon}=\operatorname{Ad}_{\exp (-\varepsilon B)} \omega^{\mathcal{H}} \circ T \varsigma+\hat{\omega} \circ T(h \exp (\varepsilon B)),
$$

where $\hat{\omega}: T_{h \exp (\varepsilon B)} H \rightarrow \mathfrak{h}, \hat{\omega}=T L_{(h \exp (\varepsilon B))^{-1}}$, stands for the (left) Maurer-Cartan form. From (4.4) and (4.5) we obtain

$$
\begin{aligned}
\omega^{\mathcal{H}} \circ T s_{\varepsilon}= & \operatorname{Ad}_{\exp (-\varepsilon B)} \omega^{\mathcal{H}} \circ T \varsigma+T L_{\exp (-\varepsilon B) h^{-1}} \\
& \circ\left(T L_{h} \circ T \exp (\varepsilon B)+T R_{\exp (\varepsilon B)} \circ T h\right) \\
= & \operatorname{Ad}_{\exp (-\varepsilon B)} \omega^{\mathcal{H}} \\
& \circ T \varsigma+T L_{\exp (-\varepsilon B)} \circ T \exp (\varepsilon B)+\operatorname{Ad}_{\exp (-\varepsilon B)} \circ T L_{h^{-1}} \circ T h .
\end{aligned}
$$

Therefore, for $x \in M$ we get

$$
\begin{aligned}
\sigma_{\varepsilon}(x) & =\tilde{\omega}^{\mathcal{H}} \circ T_{x} s_{\varepsilon}=\left\{s(x) \exp (\varepsilon B), \omega^{\mathcal{H}} \circ T_{x} s_{\varepsilon}\right\}_{H}=\left\{s(x), \operatorname{Ad}_{\exp (\varepsilon B)} \circ \omega^{\mathcal{H}} \circ T_{x} s_{\varepsilon}\right\}_{H} \\
& =\left\{s, \omega^{\mathcal{H}} \circ T \varsigma+\operatorname{Ad}_{\exp (\varepsilon B)} T L_{\exp (-\varepsilon B)} \circ T \exp (\varepsilon B)+T L_{h^{-1}} \circ T h\right\}_{H} .
\end{aligned}
$$

The infinitesimal variation $\delta \sigma$ is the derivative of the previous formula with respect to $\varepsilon$. If we interpret this vector field as a new section of $T^{*} M \otimes \tilde{\mathfrak{h}}$ (see (4.2) above), we do not need to take the derivative of the first component of $\sigma_{\varepsilon}$ as an equivalence class $\{,\}_{H}$. Thus we get

$$
\begin{aligned}
\delta \sigma & =\left.\frac{d}{d \varepsilon}\right|_{\varepsilon=0} \sigma_{\varepsilon}=\left\{s,\left.\frac{d}{d \varepsilon}\right|_{\varepsilon=0} \operatorname{Ad}_{\exp (\varepsilon B)} \circ T L_{\exp (-\varepsilon B)} \circ T \exp (\varepsilon B)\right\}_{H} \\
& =\{s, \mathrm{~d} B\}_{H}=\left\{s, \mathrm{~d} B+\left[\omega^{\mathcal{H}} \circ T s, B\right]-\left[\omega^{\mathcal{H}} \circ T s, B\right]\right\}_{H}=\nabla^{\mathcal{H}} \eta-[\sigma, \eta],
\end{aligned}
$$

since the covariant derivative of the linear connection on $\varsigma^{*} \tilde{\mathfrak{h}}$ when applied to the section $\eta=\{s, B\}_{H}$ equals $\nabla^{\mathcal{H}} \eta=\left\{s, \mathrm{~d} B+\left[\omega^{\mathcal{H}} \circ T s, B\right]\right\}_{H}$, as a direct verification (that uses the definition of the induced covariant derivative) shows. 
Remark 7. Since $\eta \in \Gamma\left(\varsigma^{*} \tilde{\mathfrak{h}}\right)$, its covariant differential as well as its Lie bracket with $\sigma \in \Gamma\left(T^{*} M \otimes \tilde{\mathfrak{h}}\right)$ yields a section of $\pi_{M \Sigma}^{*} T^{*} M \otimes_{P / H} \tilde{\mathfrak{h}} \equiv T^{*} M \otimes \tilde{\mathfrak{h}}$. Then formula (4.3) makes sense as it represents an equality in the set of sections of this bundle, if we understand $\delta \sigma$ as in (4.2).

4.3. Horizontal variations. We now consider horizontal variations with respect to $\mathcal{H}$, that is, infinitesimal variations $\delta s$ along a given section $s$ such that $\omega^{\mathcal{H}}(\delta s)=0$. In this case, if we take a variation $s_{\varepsilon}$ defining $\delta s$, the sections $\varsigma_{\varepsilon}=\left\{s_{\varepsilon}\right\}_{H}$ of $P / H \rightarrow M$ are not constant and define a vector field $\delta \varsigma$ along $\varsigma$. The variation $\delta \sigma$ along the section $\sigma=\tilde{\omega}^{\mathcal{H}} \circ T s$ of the composite bundle $\pi_{M \Sigma}^{*} T^{*} M \otimes_{P / H} \tilde{\mathfrak{h}} \equiv T^{*} M \otimes \tilde{\mathfrak{h}} \rightarrow M$ projects to $\delta \varsigma$ on $P / H$. The connection $\mathcal{H}$ on $P \rightarrow P / H$ defines a connection on its associated bundle $\tilde{\mathfrak{h}} \rightarrow P / H$ and, with the aid of the trivial connection on $T^{*} M \rightarrow P / H$, it induces a connection on the tensor product $\pi_{M \Sigma}^{*} T^{*} M \otimes_{P / H} \tilde{\mathfrak{h}} \equiv T^{*} M \otimes \tilde{\mathfrak{h}}$. Then $\delta \sigma$ can be decomposed in the vertical and horizontal parts $\delta \sigma=(\delta \sigma)_{v}+(\delta \sigma)_{h}$ with respect to this connection. As $\delta \sigma$ projects to $\delta \varsigma$, it is clear that $(\delta \sigma)_{h}$ is the horizontal lift $\operatorname{Hor}(\delta \varsigma)$ of $\delta \zeta$. The vector field

$$
(\delta \sigma)_{v}=\delta \sigma-\operatorname{Hor}(\delta \varsigma)
$$

is vertical in the vector bundle $T^{*} M \otimes \tilde{\mathfrak{h}} \rightarrow P / H$ and can thus be understood as a section of $T^{*} M \otimes \tilde{\mathfrak{h}} \rightarrow M$, as we have done in Sect. 4.2 for formula (4.2).

Proposition 5. With the notation given above, we have

$$
(\delta \sigma)_{v}=\tilde{\Omega}^{\mathcal{H}}(\delta \varsigma, T \varsigma)
$$

where $\tilde{\Omega}^{\mathcal{H}}$ is the curvature of $\mathcal{H}$, seen as a $\tilde{\mathfrak{h}}$-valued 2-form on $P / H$.

Proof. The definition of the vertical part of $\delta \sigma$ gives

$$
(\delta \sigma)_{v}=\left.\frac{d}{d \varepsilon}\right|_{\varepsilon=0} \sigma_{\varepsilon}-\operatorname{Hor}\left(\delta\{s\}_{H}\right) .
$$

The horizontal lift of the vector $\delta\{s\}_{H}=\left.\frac{d}{d \varepsilon}\right|_{\varepsilon=0}\left\{s_{\varepsilon}\right\}$ to $\tilde{\mathfrak{h}}$ is the image via the projection $P \times \mathfrak{h} \rightarrow(P \times \mathfrak{h}) / G=\tilde{h}$ of its horizontal lift to $P$ with respect to $\mathcal{H}$, which is $\delta s=d s_{\varepsilon} / d \varepsilon$, since the variation is horizontal. Then the horizontal lift at all the points of the form $\left\{s(x), \omega^{\mathcal{H}} \circ T_{x} s\right\}_{H}, x \in M$, is

$$
\operatorname{Hor}\left(\delta\{s\}_{H}\right)=\left.\frac{d}{d \varepsilon}\right|_{\varepsilon=0}\left\{s_{\varepsilon}, \omega^{\mathcal{H}} \circ T s\right\}_{H} .
$$

As the formula we want to prove is local and every principal bundle is locally trivial, it is enough to consider the special case $P=P / H \times H$. For such a trivial bundle, every section $s$ can be written as $s(x)=(\varsigma(x), h(x))$ for a certain mapping $h: M \rightarrow H$. The adjoint bundle $\tilde{\mathfrak{h}} \rightarrow P / H$ is then identified with $P / H \times \mathfrak{h}$ by putting

$$
\{(\varsigma, h), B\}_{H} \mapsto\left(\varsigma, \operatorname{Ad}_{h} B\right) .
$$


Using these identifications, from (4.6) and (4.7), we obtain

$$
\begin{aligned}
(\delta \sigma)_{v} & =\left.\frac{d}{d \varepsilon}\right|_{\varepsilon=0}\left\{s_{\varepsilon}, \omega^{\mathcal{H}} \circ T s_{\varepsilon}\right\}_{H}-\left.\frac{d}{d \varepsilon}\right|_{\varepsilon=0}\left\{s_{\varepsilon}, \omega^{\mathcal{H}} \circ T s\right\}_{H} \\
& =\left.\frac{d}{d \varepsilon}\right|_{\varepsilon=0}\left(\varsigma_{\varepsilon}, \operatorname{Ad}_{h_{\varepsilon}} \circ \omega^{\mathcal{H}} \circ T s_{\varepsilon}\right)-\left.\frac{d}{d \varepsilon}\right|_{\varepsilon=0}\left(\varsigma_{\varepsilon}, \operatorname{Ad}_{h_{\varepsilon}} \circ \omega^{\mathcal{H}} \circ T s\right) \\
& =\left(\varsigma,\left.\operatorname{Ad}_{h} \circ \frac{d}{d \varepsilon}\right|_{\varepsilon=0} \omega^{\mathcal{H}} \circ T s_{\varepsilon}\right),
\end{aligned}
$$

where for the last step, we make use of the chain rule. Then

$$
\begin{aligned}
(\delta \sigma)_{v} & =\left(\varsigma,\left.\operatorname{Ad}_{h} \circ \frac{d}{d \varepsilon}\right|_{\varepsilon=0} \omega^{\mathcal{H}} \circ T s_{\varepsilon}\right) \\
& =\left\{s,\left.\frac{d}{d \varepsilon}\right|_{\varepsilon=0} \omega^{\mathcal{H}} \circ T s_{\varepsilon}\right\}_{H} \\
& =\left\{s, \mathrm{~d} \omega^{\mathcal{H}}(\delta s, T s)\right\}_{H},
\end{aligned}
$$

as $\delta s=d s_{\varepsilon} /\left.d \varepsilon\right|_{\varepsilon=0}$. From the Cartan formula $\Omega^{\mathcal{H}}=\mathrm{d} \omega^{\mathcal{H}}+\left[\omega^{\mathcal{H}}, \omega^{\mathcal{H}}\right]$, as $\delta s$ is horizontal, we have $\mathrm{d} \omega^{\mathcal{H}}(\delta s, T s)=\Omega(\delta s, T s)$. The proof is complete by taking into account the definition of $\tilde{\Omega}(\delta \varsigma, T \varsigma)(c f .[14, \mathrm{p} .76])$.

Corollary 1. Let $s_{\varepsilon}$ be an arbitrary variation of a section $s$ of $\pi_{M P}$, with infinitesimal variation $\delta$ s. Then the infinitesimal projected variation on $\left(J^{1} P\right) / H=J^{1}(P / H) \times_{P / H}$ $\left(T^{*} M \otimes \tilde{\mathfrak{h}}\right)$ is

$$
\left.\frac{d}{d \varepsilon}\right|_{\varepsilon=0}\left(j^{1} \varsigma_{\varepsilon}, \sigma_{\varepsilon}\right)=\left(\left.\frac{d}{d \varepsilon}\right|_{\varepsilon=0} j^{1} \varsigma_{\varepsilon}, \nabla^{\mathcal{H}} \eta-[\sigma, \eta]+\tilde{\Omega}^{\mathcal{H}}(\delta \varsigma, T \varsigma)\right) .
$$

Proof. The connection $\mathcal{H}$ decomposes the infinitesimal variation $\delta s$ in the vertical and horizontal components: $\delta s=\delta^{v} s+\delta^{h} s$. From Proposition 4, for the first component we obtain $\delta \zeta=0$ and $\delta \sigma=\nabla^{\mathcal{H}} \eta+[\sigma, \eta]$. On $J^{1}(P / H) \times{ }_{P / H}\left(T^{*} M \otimes \tilde{\mathfrak{h}}\right)$ the infinitesimal variation will have the same expression $\left(0, \nabla^{\mathcal{H}} \eta+[\sigma, \eta]\right)$. Now, for the horizontal component, Proposition 5 gives $\delta \sigma=\tilde{\Omega}^{\mathcal{H}}(\delta \varsigma, T \varsigma)+\operatorname{Hor}(\delta \varsigma)$. Then, on the fiber product $J^{1}(P / H) \times_{P / H}\left(T^{*} M \otimes \tilde{\mathfrak{h}}\right)$, the infinitesimal variation will be $\left(d /\left.d \varepsilon\right|_{\varepsilon=0} j^{1} \varsigma_{\varepsilon}, \tilde{\Omega}^{\mathcal{H}}(\delta \varsigma, T \varsigma)\right)$ as both vector fields $\operatorname{Hor}(\delta \varsigma)$ and $d /\left.d \varepsilon\right|_{\varepsilon=0} j^{1} \varsigma_{\varepsilon}$ project to $\delta \varsigma$. Adding up the horizontal and the vertical contributions, we obtain formula (4.8).

Remark 8 . Corollary 1 clearly shows that the reduced variational principle defined by $L$ on the quotient $J^{1}(P / H) \times_{P / H}\left(T^{*} M \otimes \tilde{\mathfrak{h}}\right)$ is not free. From the expression (4.8), we note that not every possible variation $\delta \sigma$ comes from a variation $\delta s$, i.e., the variations of the reduced variational problem have constraints. For example, for $H=G=\mathbb{R}$, $P=M \times G$, and $\mathcal{H}$ the trivial connection, we have $\pi_{M \Sigma}^{*} T^{*} M \otimes_{P / H} \tilde{\mathfrak{h}} \equiv T^{*} M \otimes \tilde{\mathfrak{g}} \simeq$ $T^{*} M$, i.e., $\sigma$ and $\delta \sigma$ can be seen as forms on $M$. Formula (4.8) simply reads $\delta \sigma=\mathrm{d} \eta$, for an arbitrary $\eta \in C^{\infty}(M)$, that is, only those variations which are exact forms are admissible, which represents a non trivial topological constraint. 


\subsection{Notations}

4.4.1. The operator $\delta l / \delta \sigma$. Let $l: J^{1}(P / H) \times{ }_{P / H}\left(T^{*} M \otimes \tilde{\mathfrak{h}}\right) \rightarrow \mathbb{R}$ be a smooth function and let $\sigma: M \rightarrow \pi_{M \Sigma}^{*} T^{*} M \otimes P / H \tilde{\mathfrak{h}} \equiv T^{*} M \otimes \tilde{\mathfrak{h}}$ be a section of the composite fiber bundle. We define $\delta l / \delta \sigma$ as the vertical derivative of $l$ along $\sigma$ on the component $T^{*} M \otimes \tilde{\mathfrak{h}}$. More precisely, given a tangent vector $Y$ at $\sigma(x)$, vertical with respect to the projection $T^{*} M \otimes \tilde{\mathfrak{h}} \rightarrow P / H$, we define

$$
\left\langle\frac{\delta l}{\delta \sigma}, Y\right\rangle:=\left.\frac{d}{d \varepsilon}\right|_{\varepsilon=0} l\left(j_{x}^{1} \varsigma, \sigma(x)+\varepsilon Y\right) .
$$

Since $T^{*} M \otimes \tilde{\mathfrak{h}} \rightarrow P / H$ is a vector bundle, $Y$ can be thought of as an element of the fiber over $\zeta(x) \in P / H$. Then, $\delta l / \delta \sigma$ can be interpreted as a section of the dual composite fiber bundle $\left(T^{*} M \otimes \tilde{\mathfrak{h}}\right)^{*}=T M \otimes \tilde{\mathfrak{h}}^{*} \rightarrow P / H \rightarrow M$ which projects on the same section $\varsigma: M \rightarrow P / H$ as $\sigma$.

4.4.2. The coadjoint operator. The fiberwise bracket operation ad: $\tilde{\mathfrak{h}} \oplus_{P / H} \tilde{\mathfrak{h}} \rightarrow \tilde{\mathfrak{h}}$ defined on the adjoint bundle $\tilde{\mathfrak{h}}$, induces in a natural way a morphism (the coadjoint morphism) $\operatorname{ad}^{*}: \tilde{\mathfrak{h}} \oplus_{P / H} \tilde{\mathfrak{h}}^{*} \rightarrow \tilde{\mathfrak{h}}^{*},(\alpha, \mu) \mapsto \operatorname{ad}_{\alpha}^{*} \mu$, by $\left\langle\operatorname{ad}_{\alpha}^{*} \mu, \beta\right\rangle:=\left\langle\mu, \operatorname{ad}_{\alpha} \beta\right\rangle$, for $\alpha, \beta \in \tilde{\mathfrak{h}}_{y}, \mu \in \tilde{\mathfrak{h}}_{y}^{*}, y \in P / H$, where $\langle$,$\rangle denotes the natural pairing between \tilde{\mathfrak{h}}$ and $\tilde{\mathfrak{h}}^{*}$. This operator can be generalized in a natural way to a morphism

$$
\mathrm{ad}^{*}:\left(T^{*} M \otimes \tilde{\mathfrak{h}}\right) \oplus P / H\left(T M \otimes \tilde{\mathfrak{h}}^{*}\right) \rightarrow \tilde{\mathfrak{h}}^{*},
$$

where by abuse of notation, we shall also call it ad*. Fiberwise, this morphism is defined as follows. Let $y \in P / H$ and $\sigma \in\left(T^{*} M \otimes \tilde{\mathfrak{h}}\right)_{y}$ be of the form $\sigma=\omega \otimes \eta$, with $\omega \in T_{x}^{*} M$, $\pi_{M \Sigma}(y)=x, \eta \in \tilde{\mathfrak{h}}_{y}$. For $\mathcal{X} \in(T M \otimes \tilde{\mathfrak{h}})_{y}$ of the form $\mathcal{X}=X \otimes \theta, X \in T_{x} M, \theta \in \tilde{\mathfrak{h}}_{y}^{*}$, we define

$$
\operatorname{ad}_{\sigma}^{*} \mathcal{X}:=\left(i_{X} \omega\right) \operatorname{ad}_{\eta}^{*} \theta
$$

that is, the usual coadjoint operator combined with the natural pairing between $T M$ and $T^{*} M$. This definition can be generalized to arbitrary elements of $\pi_{M \Sigma}^{*} T^{*} M \otimes_{P / H} \tilde{\mathfrak{h}} \equiv$ $T^{*} M \otimes \tilde{\mathfrak{h}}$ and its dual $T M \otimes \tilde{\mathfrak{h}}^{*}$ by linearity.

4.4.3. The $\operatorname{div}^{\mathcal{H}}$ operator. Let $\nabla^{* \mathcal{H}}: \Omega^{s}\left(P / H, \tilde{\mathfrak{h}}^{*}\right) \rightarrow \Omega^{s+1}\left(P / H, \tilde{\mathfrak{h}}^{*}\right), s \in \mathbb{N}$, be the covariant differential defined by the connection $\mathcal{H}$ on the space of $\tilde{\mathfrak{h}}^{*}$-valued forms on $P / H$. We consider the volume form $v$ on $M$ and its pull-back $\pi_{M \Sigma}^{*} v \in \Omega^{n}(P / H)$, which will be denoted also by $v$, for sake of simplicity. Now let $\mathcal{X}$ be a section of the bundle $T M \otimes \tilde{\mathfrak{h}}^{*} \rightarrow M$ and $i_{\mathcal{X}} v \in \Omega^{n-1}\left(M, \tilde{\mathfrak{h}}^{*}\right) \subset \Omega^{n-1}\left(P / H, \tilde{\mathfrak{h}}^{*}\right)$ its contraction with $v$. It is clear that there exists a unique section $\operatorname{div}^{\mathcal{H}} \mathcal{X}$ of the bundle $\tilde{\mathfrak{h}}^{*} \rightarrow M$ such that

$$
\nabla^{* \mathcal{H}} i \mathcal{X} v=v \otimes \operatorname{div}^{\mathcal{H}} \mathcal{X}
$$

In this way, we can define an operator

$$
\operatorname{div}^{\mathcal{H}}: \Gamma\left(T M \otimes \tilde{\mathfrak{h}}^{*}\right) \rightarrow \Gamma\left(\tilde{\mathfrak{h}}^{*}\right),
$$


which generalizes the ordinary divergence operator div $: \Gamma(T M) \rightarrow \mathbb{R}$ for vector fields, to vector bundle valued vector fields. It is easy to check that for any section $\eta$ of $\tilde{\mathfrak{h}}$ the following formula holds:

$$
\operatorname{div}(\langle\mathcal{X}, \eta\rangle)=\left\langle\operatorname{div}^{\mathcal{H}} \mathcal{X}, \eta\right\rangle+\left\langle\mathcal{X}, \nabla^{\mathcal{H}} \eta\right\rangle
$$

We refer the reader to [4] for a proof of this property and for a local expression of $\operatorname{div}^{\mathcal{H}}$.

4.4.4. The partial Euler-Lagrange operator. Given a function $l: J^{1}(P / H) \times{ }_{P / H}$ $\left(T^{*} M \otimes \tilde{\mathfrak{h}}\right) \rightarrow \mathbb{R}$ and a section $\sigma: M \rightarrow T^{*} M \otimes \tilde{\mathfrak{h}}$ of the composite fiber bundle, we define the restricted function

$$
l_{\varsigma}: J^{1}(P / H) \rightarrow \mathbb{R}, \quad l_{\varsigma}\left(j_{x}^{1} \varsigma\right):=l\left(j_{x}^{1} \varsigma, \sigma(x)\right)
$$

Then, by definition, the partial Euler-Lagrange operator $\mathcal{E} \mathcal{L}_{\zeta}(l)$ at a section $\sigma$ is the standard Euler-Lagrange operator of the restricted function $l_{\varsigma}$ along the section $j^{1} \varsigma$.

4.5. Reducing the variational problem. The goal of this section is to present how the variational problem defined by $L$ projects to $\left(J^{1} P\right) / H$ and what properties a reduced section must satisfy in order to be the projection of a critical section of the original problem. With the notations introduced up to now, this result can be stated as follows.

Theorem 1 (Lagrange-Poincaré reduction). Let $\pi_{M P}: P \rightarrow M$ be a $G$-principal bundle over a compact manifold $M$ with volume form $v$. Let $L: J^{1} P \rightarrow \mathbb{R}$ be a Lagrangian which is invariant under the action of a subgroup $H \subset G$. We fix a principal connection $\mathcal{H}$ (with connection one-form $\omega^{\mathcal{H}}$ ) on the bundle $P \rightarrow P / H$. Let $l: J^{1}(P / H) \times_{P / H}\left(T^{*} M \otimes \tilde{\mathfrak{h}}\right) \rightarrow \mathbb{R}$ be the mapping defined by $L$ on the quotient by means of the identification (3.2). If $s$ is a section of $\pi_{M P}$, we define the section $\sigma$ of the composite bundle $T^{*} M \otimes \tilde{\mathfrak{h}} \rightarrow M$ by $\sigma(x)=\left\{s(x), \omega^{\mathcal{H}} \circ T_{x} s\right\}_{H}$ and the section $\varsigma$ of the bundle $P / H \rightarrow M$ by $\varsigma(x)=\{s(x)\}_{H}$ for $x \in M$. Then the following are equivalent:

(1) the variational principle

$$
\delta \int_{M} L\left(j^{1} s\right) v=0
$$

holds for arbitrary variations $\delta$;

(2) s satisfies the Euler-Lagrange equations for $L v$;

(3) the variational principle

$$
\delta \int_{M} l\left(j^{1} \varsigma, \sigma\right)=0,
$$

holds, for variations of the form $\delta \sigma=\nabla^{\mathcal{H}} \eta-[\sigma, \eta]+\tilde{\Omega}^{\mathcal{H}}(\delta \varsigma, T \varsigma)$, where $\delta \varsigma$ is an arbitrary variation of $\varsigma$ and $\eta$ is an arbitrary section of $\varsigma^{*} \tilde{\mathfrak{h}}$; 
(4) $\sigma$ satisfies the Lagrange-Poincaré equations

$$
\begin{gathered}
\mathcal{E} \mathcal{L}_{\zeta}(l)=\left\langle\frac{\delta l}{\delta \sigma}, i_{T \varsigma} \tilde{\Omega}^{\mathcal{H}}\right\rangle, \\
\operatorname{div}^{\mathcal{H}} \frac{\delta l}{\delta \sigma}+\operatorname{ad}_{\sigma}^{*} \frac{\delta l}{\delta \sigma}=0
\end{gathered}
$$

where $\langle$,$\rangle represents the natural pairing between \pi_{M \Sigma}^{*} T^{*} M \otimes_{P / H} \tilde{\mathfrak{h}} \equiv T M \otimes \tilde{\mathfrak{h}}^{*}$ and its dual $T M^{*} \otimes \tilde{\mathfrak{h}}$.

Note that $i_{T \varsigma} \tilde{\Omega}^{\mathcal{H}}$ is a section of $T^{*}(P / H) \otimes\left(T^{*} M \otimes \tilde{\mathfrak{h}}\right)$. After the paring with $\delta l / \delta \sigma$ we have a 1 -form on $P / H$. As $\mathcal{E} \mathcal{L}_{\varsigma}(l)$ is a vertical 1 -form on $P / H$, that is, a 1-form for vertical vectors with respect to the projection $\pi_{M \Sigma}: P / H \rightarrow M$, one has to consider the restriction of $\left\langle\delta l / \delta \sigma, i_{T} \tilde{\Omega}^{\mathcal{H}}\right\rangle$ to vertical vectors in order to have an identity of vertical 1-forms in the first equation of (4.11).

Proof. The equivalence $(1) \Leftrightarrow(2)$ is the classical result of the calculus of variations. For $(1) \Leftrightarrow(3)$, we know that, given a variation $s_{\varepsilon}$,

$$
\left.\frac{d}{d \varepsilon}\right|_{\varepsilon=0} \int_{M} L\left(j^{1} s_{\varepsilon}\right) v=\left.\frac{d}{d \varepsilon}\right|_{\varepsilon=0} \int l\left(j^{1} \varsigma_{\varepsilon}, \sigma_{\varepsilon}\right) v .
$$

By virtue of Corollary 1 , we have

$$
\left.\frac{d}{d \varepsilon}\right|_{\varepsilon=0}\left(j^{1} \varsigma_{\varepsilon}, \sigma_{\varepsilon}\right)=\left(\left.\frac{d}{d \varepsilon}\right|_{\varepsilon=0} j^{1} \varsigma_{\varepsilon}, \nabla^{\mathcal{H}} \eta-[\sigma, \eta]+\tilde{\Omega}^{\mathcal{H}}(\delta \varsigma, T \varsigma)\right) .
$$

Then $\delta \int_{M} L\left(j^{1} s\right) v=0$ if and only if $\delta \int_{M} l\left(j^{1} \varsigma, \sigma\right) v=0$ with respect to this type of variations. Finally, for $(3) \Leftrightarrow(4)$, we write

$$
\begin{gathered}
\left.\frac{d}{d \varepsilon}\right|_{\varepsilon=0} \int_{M} l\left(j^{1} \varsigma_{\varepsilon}, \sigma_{\varepsilon}\right) v=\int_{M}\left(\frac{\delta l}{\delta \sigma}(\delta \sigma)+\frac{\delta l}{\delta \varsigma}\left(\left.\frac{d}{d \varepsilon}\right|_{\varepsilon=0} j^{1} \varsigma_{\varepsilon}\right)\right) v \\
=\int_{M}\left(\frac{\delta l}{\delta \sigma}\left(\nabla^{\mathcal{H}} \eta-[\sigma, \eta]+\tilde{\Omega}^{\mathcal{H}}(\delta \varsigma, T \varsigma)\right)+\left\langle\mathcal{E} \mathcal{L}_{\varsigma}(l), \delta \varsigma\right\rangle\right) v,
\end{gathered}
$$

where $\delta l / \delta \varsigma$ yields its adjoint operator, that is, the partial Euler-Lagrange operator $\mathcal{E} \mathcal{L}_{\zeta}(l)$, by integration by parts. Then, from formula (4.10), we have

$$
\begin{aligned}
\int_{M}\left\langle\frac{\delta l}{\delta \sigma}, \nabla^{\mathcal{H}} \eta\right\rangle v & =\int_{M}\left(\operatorname{div}\left\langle\frac{\delta l}{\delta \sigma}, \eta\right\rangle-\left\langle\operatorname{div}^{\mathcal{H}}\left(\frac{\delta l}{\delta \sigma}\right), \eta\right\rangle\right) v \\
& =-\int_{M}\left\langle\operatorname{div}^{\mathcal{H}}\left(\frac{\delta l}{\delta \sigma}\right), \eta\right\rangle v
\end{aligned}
$$

because $\int_{M} \operatorname{div}\langle\delta l / \delta \sigma, \eta\rangle v=0$ by Stokes' Theorem. We thus obtain

$\delta \int_{M} l\left(j^{1} \varsigma, \sigma\right) v=\int_{M}\left(\left\langle-\operatorname{div}^{\mathcal{H}} \frac{\delta l}{\delta \sigma}-\operatorname{ad}_{\sigma}^{*} \frac{\delta l}{\delta \sigma}, \eta\right\rangle+\left\langle\mathcal{E} \mathcal{L}_{\varsigma}(l)-\left\langle\frac{\delta l}{\delta \sigma}, i_{{ }^{*}} \tilde{\Omega}^{\mathcal{H}}\right\rangle, \delta \varsigma\right\rangle\right) v$,

and then, from the fundamental lemma of the calculus of variations, $\eta$ and $\delta \varsigma$ being arbitrary, we conclude that $\delta \int_{M} l\left(j^{1} \varsigma, \sigma\right) v=0$ holds if and only if the Lagrange-Poincaré equations are satisfied. 


\section{Compatibility Conditions and Reconstruction}

Given a critical section $s: M \rightarrow P$ of the variational problem defined by $L v$ we obtain a reduced section $\sigma: M \rightarrow T^{*} M \otimes \tilde{\mathfrak{h}}$ which is a solution of the Lagrange-Poincaré equations for $l$; recall that we use the notation $\pi_{M \Sigma}^{*} T^{*} M \otimes_{P / H} \tilde{\mathfrak{h}} \equiv T^{*} M \otimes \tilde{\mathfrak{h}}$. In Sect. 3.2 we showed that $\sigma$ defines two geometrical objects: a $H$-reduction $P^{\varsigma}=\pi_{\Sigma P}^{-1}(\varsigma(M))$ of the bundle $P$ and a connection $\mathcal{H}^{\sigma}$ on it. In fact, the relationship between $P^{\varsigma}$ and $s$ is clear: the bundle $P^{\varsigma}$ is the $H$-orbit of the range $s(M)$, that is, $P^{\varsigma}=\left\{R_{h}(s(x)) \mid x \in\right.$ $M, h \in H\} \subset P$.

The bundle $P^{\varsigma}$ is endowed with the natural flat connection defined by the foliation $\left\{R_{h}(s(M))\right\}_{h \in H}$, i.e. the flat connection whose integrable leaves are the sets $\left\{R_{h}(s(x)) \mid\right.$ $x \in M\}, h \in H$, that is, copies of the range of $s$ for every group element $h \in H$. We now check that this connection is nothing but the connection $\mathcal{H}^{\sigma}$. By definition (see Sect. 3.2), the connection one-form of $\mathcal{H}^{\sigma}$ at a point $s(x) \in P^{\varsigma}$ is

$$
\omega:=\omega^{\mathcal{H}}-\omega^{\mathcal{H}} \circ T s \circ T \pi_{M P}=\omega^{\mathcal{H}} \circ\left(\mathrm{Id}-T\left(s \circ \pi_{M P}\right)\right),
$$

which is exactly the one-form defined by the decomposition $T_{s(x)} P=T_{x} s\left(T_{x} M\right) \oplus \mathfrak{h}_{s(x)}^{*}$ induced by the above mentioned foliation on $P^{5}$; recall that $\mathfrak{h}_{p}^{*}:=\left\{B_{p}^{*} \mid B \in \mathfrak{h}\right\}$. Therefore, $\mathcal{H}^{\sigma}$ is a flat connection. This imposes a necessary condition on the section $\sigma$ of $T^{*} M \otimes \tilde{\mathfrak{h}}$ to be the projection of a section $s$ of $P \rightarrow M$, namely, the curvature of $\mathcal{H}^{\sigma}$ must vanish. Thus, an arbitrary solution of the Lagrange-Poincaré equations is not always of the form $\sigma(x)=\left\{s(x), \omega^{\mathcal{H}} \circ T_{x} s\right\}_{H}$.

The condition $\operatorname{Curv}\left(\mathcal{H}^{\sigma}\right)=0$ is also sufficient, at least locally. Therefore it becomes a compatibility condition which must be imposed to reconstruct a solution $s$ from the solution $\sigma$. More precisely:

Theorem 1. A section $s: M \rightarrow P$ that is a solution of the Euler-Lagrange equations for $L$ defines a section $\sigma: M \rightarrow \pi_{M \Sigma}^{*} T^{*} M \otimes_{P / H} \tilde{\mathfrak{h}} \equiv T^{*} M \otimes \tilde{\mathfrak{h}}$ that is a solution of the Lagrange-Poincaré equations such that $\mathcal{H}^{\sigma}$ is a flat connection. Conversely, given a solution $\sigma$ of the Lagrange-Poincare equations such that $\mathcal{H}^{\sigma}$ is flat and with trivial holonomy, the family of solutions $s_{h}(x)=s(x) h, s: M \rightarrow P, h \in H$, of the original problem defined by $L$ are obtained as follows: construct the $H$-reduced bundle $P^{\zeta}$ defined by the section $5: M \rightarrow P / H$ and the integrable manifolds of the horizontal bundle of $\mathcal{H}^{\sigma}$ are the images $s_{h}(M), h \in H$, of the desired family. Roughly speaking, we have locally the equivalence

\section{$\left.\begin{array}{c}\text { Solutions s of the } \\ \text { Euler-Lagrange equations }\end{array}\right\} \Longleftrightarrow\left\{\begin{array}{c}\text { Solutions } \sigma \text { of the Lagrange-Poincaré } \\ \text { equations with } \mathcal{H}^{\sigma} \text { a flat connection. }\end{array}\right.$}

Proof. Given a critical section $s$ of the problem defined by $L$, we have already seen that the section $\sigma$ solves the Lagrange-Poincaré equations and $\mathcal{H}^{\sigma}$ is a flat connection. Conversely, given $\sigma$ such that $\operatorname{Curv}\left(\mathcal{H}^{\sigma}\right)=0$ and with trivial holonomy, the integral leaves $s$ are sections of $P^{\varsigma}$. We have $\sigma=\left\{s(x), \omega^{\mathcal{H}} \circ T S\right\}_{H}$ and by virtue of Theorem 1, $s$ is a critical section of $L$.

Remark 9. If $M$ is not simply connected, the integral leaves of a flat connection are not, in general, global sections of the bundle $\pi_{M P}: P \rightarrow M$. There are flat connections with non-trivial holonomy (cf. [14, Ch. II, Sect. 9]). Nevertheless, locally the holonomy of every flat connection is always trivial, that is, for every $x \in M$ there is a neighborhood $U$ such that the integral leaves of the flat connection on $\pi_{M P}^{-1}(U)$ are sections of $\pi_{M P}$ on $U$. 
Remark 10. It is not difficult to check that the curvature of $\mathcal{H}^{\sigma}$ can be expressed in terms of the form $\omega^{\mathcal{H}}$ and $\sigma$ in the following way:

$$
\operatorname{Curv}\left(\mathcal{H}^{\sigma}\right)=\Omega^{\mathcal{H}}-\nabla^{\mathcal{H}} \sigma+[\sigma, \sigma]
$$

where $\Omega^{\mathcal{H}}$ is the curvature of $\mathcal{H}$ and $\nabla^{\mathcal{H}}$ stands for the covariant derivative defined by $\mathcal{H}$. Then the geometrical condition of flatness of $\mathcal{H}^{\sigma}$ can be substituted by the more analytical condition $\Omega^{\mathcal{H}}-\nabla^{\mathcal{H}} \sigma+[\sigma, \sigma]=0$ along the bundle $P^{\varsigma}$.

Remark 11 (The case of Classical Mechanics). If $\operatorname{dim} M=1$, every connection is flat and the second condition on the right-hand side of the equivalence in Theorem 1 is automatically satisfied. In this case we have the direct equivalence: Euler-Lagrange $\Longleftrightarrow$ Lagrange-Poincaré.

\section{Special Cases}

The size of the subgroup $H$ of $G$ of symmetries of the Lagrangian $L$ determines the structure of the Lagrange-Poincaré equations. More precisely: the first group of equations in (4.11) represents an Euler-Lagrange operator on the bundle $P / H \rightarrow M$, whose fiber dimension is $\operatorname{dim} G-\operatorname{dim} H$; the second group of equations in (4.11) is an operator on the bundle $\varsigma^{*} \tilde{\mathfrak{h}} \rightarrow M$ whose fiber dimension is $\operatorname{dim} H$. Roughly speaking, the bigger the dimension of $H$, the bigger the second group of equations and the smaller the first group of equations in (4.11).

6.1. Euler-Poincaré reduction. In particular, if $H=G$, the first group of equations does not appear because $P / H=M$ and the projection $P / H \rightarrow M$ is the identity. This particular case of Lagrange-Poincaré equations has been studied in [4] and are usually called Euler-Poincaré equations. The geometry of this reduction is very interesting. The quotient $C=\left(J^{1} P\right) / G$ is in fact the bundle of connections $\pi_{M C}: C \rightarrow M$ of the principal bundle, that is, the bundle over $M$ whose sections $\sigma: M \rightarrow C$ represent connections on $P \rightarrow M$ (see, for example, [3, 9, Sect. 2.7]). The bundle $C \rightarrow P$ is an affine bundle modeled over the vector bundle $T^{*} M \otimes \tilde{\mathfrak{g}}$ and the fibration $\pi_{C J}: J^{1} P \rightarrow C$ is a $G$-principal bundle.

Then, the bundle of connections is the configuration bundle of the reduced problem and the Euler-Poincaré equations are equations on connections. More precisely, Theorem 1 reads in this case as follows:

Theorem 2 (Euler-Poincaré reduction). Let $\pi: P \rightarrow M$ be a principal $G$-fiber bundle over a manifold $M$ with a volume form $v$ and let $L: J^{1} P \rightarrow \mathbb{R}$ be a $G$ invariant Lagrangian. Let $l: C \rightarrow \mathbb{R}$ be the mapping defined by $L$ on the quotient. For a section $s: M \rightarrow P$ of $\pi$, let $\sigma: M \rightarrow C$ be defined by $\sigma(x)=\pi_{C J}\left(j_{x}^{1} s\right)$. Then, for every connection $\mathcal{H}$ of the bundle $\pi$, the following are equivalent:

(1) s satisfies the Euler-Lagrange equations for $L$,

(2) the variational principle

$$
\delta \int_{M} L\left(j_{x}^{1} s\right) d x=0
$$

holds, for variations with compact support, 
(3) the Euler-Poincaré equations hold:

$$
\operatorname{div} \mathcal{H} \frac{\delta l}{\delta \sigma}+\operatorname{ad}_{\sigma \mathcal{H}}^{*} \frac{\delta l}{\delta \sigma}=0
$$

where $\sigma^{\mathcal{H}} \in \Gamma\left(T^{*} M \otimes \tilde{\mathfrak{g}}\right)$ is such that $\sigma=\mathcal{H}+\sigma^{\mathcal{H}}$,

(4) the variational principle

$$
\delta \int_{M} l(\sigma(x)) d x=0
$$

holds, using variations of the form

$$
\delta \sigma=\nabla^{\mathcal{H}} \eta-\left[\sigma^{\mathcal{H}}, \eta\right]
$$

where $\eta: M \rightarrow \tilde{\mathfrak{g}}$ is an arbitrary section.

For reconstructing, the compatibility condition in this case is $\operatorname{simply} \operatorname{Curv}(\sigma)=0$.

6.2. The discrete case. The other special case is when $H=\{e\}$ or $H$ is a discrete subgroup of $G$. Then $\tilde{\mathfrak{h}}=P / H$, the projection $\pi_{\Sigma \mathfrak{h}}: \tilde{\mathfrak{h}} \rightarrow P / H$ is the identity and every connection of the principal bundle $P \rightarrow P / H$ is flat. The second group of LagrangePoincaré equations (4.11) do not appear, while the first group reads $\mathcal{E} \mathcal{L}(l)=0$, that is, we obtain the Euler-Lagrange equations for $l$. This result is clear because the bundles $P \rightarrow M$ and $P / H \rightarrow M$ are in this case locally diffeomorphic and the Euler-Lagrange operator is a local object. The reduction by a discrete group does not change the structure of the equations but only the configuration bundle.

\section{Semidirect Product Reduction}

Sometimes, when the symmetry group $H$ of a Lagrangian $L^{\prime}: J^{1} P \rightarrow \mathbb{R}$ fails to be the entire group $G$, we can introduce a new variable $a \in N, N$ being a manifold where the group $G$ acts, and a new function $L: J^{1} P \times N \rightarrow \mathbb{R}$, such that $L$ is now $G$-invariant and $L^{\prime}\left(j_{x}^{1} s\right)=L\left(j_{x}^{1} s, a_{0}\right)$ for a fixed $a_{0} \in N$. Roughly speaking, we increase the size of the group of symmetries at the cost of a new parameter $a_{0}$. In classical mechanics, when $N$ is a vector space $V$ and the action is linear, this approach is known as the semidirect product reduction theory; see [13] for the Lagrangian case and [25, 16, 17] for the Hamiltonian case. We now extend the Lagrangian method to the field theoretical setting.

Let $G$ be a Lie group acting (on the left) on a vector space $V$ and let $L: J^{1} P \times$ $V \rightarrow \mathbb{R}$ be a function invariant under the right action $\left(j^{1} s_{x}, a_{0}\right) \cdot g=\left(\left(j_{x}^{1} s\right) g, g^{-1} a_{0}\right)$ of $G$ on $J^{1} P \times V$. For every $a_{0} \in V$, we define the Lagrangian $L_{a_{0}}: J^{1} P \rightarrow \mathbb{R}$ by $L_{a_{0}}\left(j_{x}^{1} s\right):=L\left(j_{x}^{1} s, a_{0}\right)$, that is, $L$ can be understood as a family of Lagrangians on $\pi_{M P}: P \rightarrow M$ parameterized by $a_{0} \in V$. Even though $L$ is $G$-invariant, each Lagrangian $L_{a_{0}}$ is only invariant under the isotropy subgroup $G_{a_{0}} \subset G$ of $a_{0} \in V$. In this section we are going to study the reduction of the variational problems defined by the Lagrangians $L_{a_{0}}, a_{0} \in V$, by means of the reduction of the variational problem defined by $L$ on the product $J^{1} P \times V$. 
7.1. The geometry of $\mathcal{V}=\left(J^{1} P \times V\right) / G$. If $L: J^{1} P \times V \rightarrow \mathbb{R}$ is $G$-invariant, it defines a function $l:\left(J^{1} P \times V\right) / G \rightarrow \mathbb{R}$ on the quotient. It is clear that the configuration bundle of the reduced variational problem defined by $l$ is the bundle $\left(J^{1} P \times V\right) / G \rightarrow M$ so we proceed to describe the main geometrical properties of this space. As we have said in Sect. 6.1, the projection $\pi_{C J}: J^{1} P \rightarrow C=\left(J^{1} P\right) / G$ is a principal $G$-bundle over $C$. Therefore, $\mathcal{V}=\left(J^{1} P \times V\right) / G$ is an associated vector bundle over $C$, which we are going to denote $\pi_{C} \mathcal{V}: \mathcal{V} \rightarrow C$. If $\pi_{M C}: C \rightarrow M$ denotes the bundle of connections, it is known ( $c f$. [3, 8, 9, Sect. 2.7]) that $J^{1} P$ is isomorphic to the pull-back bundle $\pi_{M C}^{*} P=C \times{ }_{M} P$ and, as a consequence, we have that $\mathcal{V}$ is isomorphic to $\pi_{M C}^{*} \mathbf{V}=C \times{ }_{M} \mathbf{V}$, where $\mathbf{V}$ is the vector bundle $\pi_{M \mathbf{V}}: \mathbf{V}=(P \times V) / G \rightarrow M$ associated to $\pi_{M P}: P \rightarrow M$ by the representation of $G$ on $V$. We can summarize these relations by means of the following commutative diagram

$$
\begin{aligned}
& \pi_{M C}^{*} P=J^{1} P \stackrel{\pi_{10}}{\longrightarrow} P \\
& \pi_{C J} \downarrow \downarrow \pi_{M P} \\
& C \stackrel{\pi_{M C}}{\longrightarrow} M \\
& \pi_{C \mathcal{V}} \uparrow \uparrow \pi_{M \mathbf{V}} \\
& \pi_{M C}^{*} \mathbf{V}=\mathcal{V} \stackrel{\pi_{\mathbf{V} V}}{\longrightarrow} \mathbf{V} .
\end{aligned}
$$

The composite bundle $\mathcal{V} \stackrel{\pi_{C} \mathcal{V}}{\rightarrow} C \stackrel{\pi_{M C}}{\rightarrow} M$ is the configuration bundle of the reduced variational problem defined by $L$. Each section $\lambda: M \rightarrow \mathcal{V}$ of this bundle gives two objects. Firstly, we obtain a section

$$
a:=\pi \mathbf{V} \mathcal{V} \circ \lambda
$$

of the vector bundle $\mathbf{V} \rightarrow M$ and, secondly, a section

$$
\sigma:=\pi_{C \mathcal{V}} \circ \lambda
$$

of the bundle of connections $C \rightarrow M$, which represents a connection $\mathcal{H}^{\sigma}$ on the principal bundle $P \rightarrow M$. If we identify $\mathcal{V}$ with $\pi_{M C}^{*} \mathbf{V}=C \times_{M} \mathbf{V}$, we can write

$$
\lambda(x)=(\sigma(x), a(x)), \quad \text { for all } \quad x \in M .
$$

7.2. The reduction of the problem defined by $L$. We now fix the value of the parameter $a_{0}$ for the study of the variational principle of the Lagrangian $L_{a_{0}}$. Let $s$ be a section of the bundle $\pi_{M P}$ and let $\lambda$ be the section of $\left(J^{1} P \times V\right) / G=\mathcal{V} \rightarrow M$ defined as $\lambda(x)=\left\{j_{x}^{1} s, a_{0}\right\}_{G}, x \in M$. From formulas (7.1) and (7.2), we obtain the sections $a$ and $\sigma$ of the bundles $C \rightarrow M$ and $\mathbf{V} \rightarrow M$ respectively. It is clear that these sections can be also defined by

$$
a(x)=\left\{s(x), a_{0}\right\}_{G}, \quad \sigma(x)=\pi_{C J}\left(j_{x}^{1} s\right) .
$$

If we identify $\mathcal{V}$ with $\pi_{M C}^{*} P=C \times_{M} \mathbf{V}$ (see Sect. 7.1 above), from formula (7.3), we have

$$
\lambda(x)=\left(\pi_{C J}\left(j_{x}^{1} s\right), a(x)\right)=(\sigma(x), a(x)), \quad x \in M .
$$


Given an infinitesimal variation $\delta s=d /\left.d \varepsilon\right|_{\varepsilon=0} s_{\varepsilon}$ of the section $s$, taking the derivative with respect to $\varepsilon$ in (7.4), we obtain an infinitesimal variation

$$
\delta \lambda=\left.\frac{d}{d \varepsilon}\right|_{\varepsilon=0} \lambda_{\varepsilon}=\left(\left.\frac{d}{d \varepsilon}\right|_{\varepsilon=0} \sigma_{\varepsilon},\left.\frac{d}{d \varepsilon}\right|_{\varepsilon=0} a_{\varepsilon}\right)=(\delta \sigma, \delta a),
$$

along the section $\lambda$. Since the vector field $\delta s$ is, by definition, vertical with respect to $\pi_{M P}$, it follows that $\delta \sigma$ and $\delta a$ are vertical with respect to $\pi_{M C}$ and $\pi_{M \mathbf{V}}$ respectively. Because $\mathbf{V} \rightarrow M$ is a vector bundle, we can think of $\delta a$ as a new section of this bundle. Similarly, since $C \rightarrow M$ is an affine bundle modeled over the vector bundle $T^{*} M \otimes \tilde{\mathfrak{g}} \rightarrow M$, we can think of $\delta \sigma$ as a section of $T^{*} M \otimes \tilde{\mathfrak{g}} \rightarrow M$.

We now give the structure of the infinitesimal variation $\delta \lambda$. We know that given $\delta s$ along $s$, there exist a mapping $B: M \rightarrow \mathfrak{g}$ such that $\delta s=B^{*}$, that is, $\delta s$ is the infinitesimal generator of the variation

$$
s_{\varepsilon}(x)=s(x) \exp (\varepsilon B(x)) .
$$

Let $\eta$ be the section of $\tilde{\mathfrak{g}} \rightarrow M$ given by $\eta(x)=\{s(x), B(x)\}_{G}$.

Proposition 6. Let $\mathcal{H}$ an arbitrary connection on the bundle $P \rightarrow M$. With a variation $s_{\varepsilon}$ as in formula (7.6) and with the notation given above, we have that

$$
\delta \sigma=\nabla^{\mathcal{H}} \eta-\left[\sigma^{\mathcal{H}}, \eta\right],
$$

where $\nabla^{\mathcal{H}}$ stands for the covariant derivative on the bundle $\tilde{\mathfrak{g}} \rightarrow M$ and $\sigma^{\mathcal{H}} \in \Gamma\left(T^{*} M \otimes\right.$ $\tilde{\mathfrak{g}})$ is such that $\sigma=\mathcal{H}+\sigma^{\mathcal{H}}$. For the variation of a we have that

$$
\delta a=\eta a,
$$

where the fiberwise action of the bundle $\tilde{\mathfrak{g}} \rightarrow M$ on $\mathbf{V} \rightarrow M$ is induced by the infinitesimal action of $\mathfrak{g}$ on $V$, that is

$$
\eta a=\{s(x), B(x)\}_{G}\left\{s(x), a_{0}\right\}_{G}=\left\{s(x), B(x) a_{0}\right\}_{G} .
$$

Proof. The expression for $\delta \sigma$ is part of Theorem 2 (Euler-Poincaré reduction). See [4] for the proof of this result. For the expression of $\delta a$, the proof is trivial by taking the derivative of

$$
a_{\varepsilon}=\left\{s_{\varepsilon}, a_{0}\right\}_{G}=\left\{s(x), \exp (\varepsilon B) a_{0}\right\}_{G}
$$

with respect to $\varepsilon$ and comparing with (7.7).

Notation. Given a section $\lambda=(\sigma, a)$ of $\mathcal{V} \rightarrow M$, we define the vertical derivatives of $l$ with respect to $\sigma$ and $a$ by

$$
\left\langle\frac{\delta l}{\delta \sigma}, Y\right\rangle:=\left.\frac{d}{d \varepsilon}\right|_{\varepsilon=0} l(\sigma(x)+\varepsilon Y, a(x)), \quad\left\langle\frac{\delta l}{\delta a}, Z\right\rangle:=\left.\frac{d}{d \varepsilon}\right|_{\varepsilon=0} l(\sigma(x), a(x)+\varepsilon Z),
$$

for every $Y \in\left(T^{*} M \otimes \tilde{\mathfrak{h}}\right)_{x}, Z \in \mathbf{V}_{x}$. Thus $\delta l / \delta \sigma$ and $\delta l / \delta a$ are sections of the dual bundles $T M \otimes \tilde{\mathfrak{h}}^{*}$ and $\mathbf{V}^{*}$ respectively.

Given an element $a_{0} \in V$, let $\rho_{a_{0}}: \mathfrak{g} \rightarrow V$ denote the linear map $\xi \mapsto \xi a_{0}$ defined by the infinitesimal Lie algebra representation induced by the action of $G$ on $V$ and let $\rho_{a_{0}}^{*}: V^{*} \rightarrow \mathfrak{g}^{*}$ the dual morphism. For notational convenience, we shall write 
$\rho_{a_{0}}^{*}\left(k_{0}\right)=a_{0} \diamond k_{0} \in \mathfrak{g}^{*}, k_{0} \in V^{*}$. This notation can be extended to the bundles $\mathbf{V}^{*}$ and $\tilde{\mathfrak{g}}^{*}$. Given a section $a \in \Gamma(\mathbf{V})$, we obtain a bundle morphism

$$
\rho_{a}: \tilde{\mathfrak{g}} \rightarrow \mathbf{V}, \quad \rho_{a}(\eta)=\eta a(x), \quad \eta \in \tilde{\mathfrak{g}}_{x}, \quad x \in M,
$$

defined in formula (7.7), and the dual morphism $\rho_{a}^{*}: \mathbf{V}^{*} \rightarrow \tilde{\mathfrak{g}}^{*}$. We shall write

$$
\rho_{a}^{*}(k)=a \diamond k \in \tilde{\mathfrak{g}}_{x}^{*}, \quad \text { for all } \quad k \in \mathbf{V}_{x}^{*}, \quad x \in M .
$$

Theorem 3 (Reduction for semidirect products). The following are equivalent:

(1) the variational principle

$$
\delta \int_{M} L_{a_{0}}\left(j_{x}^{1} s\right) v=0
$$

holds for arbitrary variations $\delta s$ of $s$,

(2) $s$ satisfies the Euler-Lagrange equations for $L_{a_{0}}$,

(3) the constrained variational principle

$$
\delta \int_{M} l(\sigma, a) v=0
$$

holds on $\mathcal{V}=C \times_{M} \mathbf{V}$, using variations of the form

$$
\delta \lambda=\nabla^{\mathcal{H}} \eta-\left[\sigma^{\mathcal{H}}, \eta\right], \quad \delta a=\eta a,
$$

where $\eta$ is an arbitrary section of the bundle $\tilde{\mathfrak{g}} \rightarrow M$,

(4) the following equation holds on $\mathcal{V}=C \times_{M} \mathbf{V}$ :

$$
\operatorname{div}^{\mathcal{H}} \frac{\delta l}{\delta \sigma}+\operatorname{ad}_{\sigma \mathcal{H}}^{*} \frac{\delta l}{\delta \sigma}=a \diamond \frac{\delta l}{\delta a} .
$$

Proof. The equivalence between (1) $\Leftrightarrow(2)$ is standard. For $(1) \Leftrightarrow(3)$, we know that, given a variation $s_{\varepsilon}$,

$$
\left.\frac{d}{d \varepsilon}\right|_{\varepsilon=0} \int_{M} L_{a_{0}}\left(j^{1} s_{\varepsilon}\right) v=\left.\frac{d}{d \varepsilon}\right|_{\varepsilon=0} \int_{M} L\left(j^{1} s_{\varepsilon}, a_{0}\right) v=\left.\frac{d}{d \varepsilon}\right|_{\varepsilon=0} \int l\left(j^{1} \sigma_{\varepsilon}, a_{\varepsilon}\right) v .
$$

Proposition 6 implies that

$$
\left.\frac{d}{d \varepsilon}\right|_{\varepsilon=0}\left(j^{1} \sigma_{\varepsilon}, a_{\varepsilon}\right)=\left(\nabla^{\mathcal{H}} \eta-[\sigma, \eta], \eta a\right)
$$

Then $\delta \int_{M} L\left(j^{1} s\right) v=0$ if and only if $\delta \int_{M} l\left(j^{1} \varsigma, \sigma\right) v=0$ with respect to this type of variations.

Finally, for $(3) \Leftrightarrow(4)$, we write

$$
\begin{aligned}
\left.\frac{d}{d \varepsilon}\right|_{\varepsilon=0} & \int_{M} l\left(j^{1} \sigma_{\varepsilon}, a_{\varepsilon}\right) v=\int_{M}\left(\frac{\delta l}{\delta \sigma}(\delta \sigma)+\frac{\delta l}{\delta a}(\delta a)\right) v \\
= & \int_{M}\left(\frac{\delta l}{\delta \sigma}\left(\nabla^{\mathcal{H}} \eta-[\sigma, \eta]\right)+\frac{\delta l}{\delta a}(\eta a)\right) v .
\end{aligned}
$$


Then, from formula (4.10), we have

$$
\begin{aligned}
\int_{M}\left\langle\frac{\delta l}{\delta \sigma}, \nabla^{\mathcal{H}} \eta\right\rangle v & =\int_{M}\left(\operatorname{div}\left\langle\frac{\delta l}{\delta \sigma}, \eta\right\rangle-\left\langle\operatorname{div}^{\mathcal{H}}\left(\frac{\delta l}{\delta \sigma}\right), \eta\right\rangle\right) v \\
& =-\int_{M}\left\langle\operatorname{div}^{\mathcal{H}}\left(\frac{\delta l}{\delta \sigma}\right), \eta\right\rangle v,
\end{aligned}
$$

since $\int_{M} \operatorname{div}\langle\delta l / \delta \sigma, \eta\rangle v=0$ by Stokes' Theorem. We thus obtain

$$
\delta \int_{M} l\left(j^{1} \varsigma, \sigma\right) v=\int_{M}\left\langle-\operatorname{div}^{\mathcal{H}} \frac{\delta l}{\delta \sigma}-\operatorname{ad}_{\sigma}^{*} \frac{\delta l}{\delta \sigma}+a \diamond \frac{\delta l}{\delta a}, \eta\right\rangle v,
$$

and then, from the fundamental lemma of the calculus of variations, $\eta$ being arbitrary, we conclude that the $\delta \int_{M} l\left(j^{1} \varsigma, \sigma\right) v=0$ if and only if (7.8) is true.

Remark 12. For $M=\mathbb{R}, P=M \times G$, we recover the classical version of the Lagrangian semidirect product reduction theory. In this case, the analogue of Theorem 3 can be found in [13, Theorem 3.3]. We remark that in [13], due to other considerations, the authors deal with $G$-invariant Lagrangians defined on $T G \times V^{*}$, that is, the space of the parameter $a_{0}$ is the dual to a vector space. For this reason, there are slight differences which must be taken into account if one wants to compare the formulation of the classical case in Theorem 3 with the statement of [13, Theorem 3.3]. For example, since the action of $\mathfrak{g}$ on $V^{*}$ is taken to be minus the dual map of the $\mathfrak{g}$-action on $V$ (the contragradiant representation), the variation of $a$ turns out to be $\delta a=-a \eta$. Moreover, for functorial convenience, we write $a \diamond \delta l / \delta a$ in the Euler-Poincaré equations (7.8) instead of $\delta l / \delta a \diamond a$, as was the case in [13, Theorem 3.3]. Apart from such formal considerations, Theorem 3 completely fits in the case of classical mechanics studied in the above mentioned paper.

7.3. Reconstruction. Given a section $s$ of the bundle $P \rightarrow M$ and a fixed element $a_{0} \in V$, we obtain sections $\sigma=\pi_{C J}\left(j^{1} s\right)$ and $a=\left\{s, a_{0}\right\}_{G}$ of $C \rightarrow M$ and $\mathbf{V} \rightarrow M$ respectively. As we have seen in Sect. 5, the range of the section $s$ is an integral leaf of the horizontal bundle of the connection $\sigma$. From this fact we deduce that $\sigma$ is a flat connection, as we have seen for the Euler-Poincaré reduction in Sect. 6.1. We now study the properties of $a$. First, it is straightforward to check that $\nabla^{\sigma} a=0$, where $\nabla^{\sigma}$ is the covariant derivative of the linear connection induced by $\sigma$ on the vector bundle $\mathbf{V} \rightarrow M$; i.e., $a$ is a flat section with respect to $\sigma$. On the other hand, for every $x \in M$, if we write $a(x)$ as $\{p, \hat{a}\}_{G}$, with $p \in \pi_{M P}^{-1}(x)$ arbitrary, it is clear that $\hat{a} \in \operatorname{Orb}\left(a_{0}\right)$, that is, if we write $a$ as an equivalence class of $(P \times V) / G$, the second term always belongs to the $G$-orbit of $a_{0}$. All these properties satisfied by $\sigma$ and $a$ are necessary conditions. An arbitrary solutions of Eqs. (7.8) is not always of the form $\sigma=\pi_{C J}\left(j^{1} s\right), a=\left\{s, a_{0}\right\}_{G}$. If the holonomy of the connection $\sigma$ is trivial (which is always true locally) these conditions are also sufficient. Therefore they become the compatibility conditions that must be imposed to reconstruct a solution $s$ of the variational problem defined by $L_{a_{0}}$ from the solutions of (7.8). More precisely:

Theorem 4. We fix a point $\hat{x} \in M$ and a value $a_{0} \in V$. With the assumptions of Theorem 3 , a solution s of the problem defined by the Lagrangian $L_{a_{0}}$ defines the sections $\sigma$ and $a$, solutions of the equations (7.8), such that $\operatorname{Curv}(\sigma)=0, \nabla^{\sigma} a=0$ and $a(\hat{x})=\{p, \hat{a}\}_{G}$, 
with $\hat{a} \in \operatorname{Orb}\left(a_{0}\right)$, for any $p \in \pi_{M P}^{-1}(x)$. Conversely, given a solution $\lambda=(\sigma, a)$ Eqs. (7.8) satisfying the previous properties, if the holonomy of $\sigma$ is trivial, the solutions of the variational problem defined by $L_{a_{0}}$ are obtained as follows. We take an integral section $\hat{s}: M \rightarrow P$ of the connection $\sigma$. We write $a(x)=\{s(x), \hat{a}\}_{G}$. Let $g$ be an element of $G$ such that $\hat{a}=g a_{0}$. Any other such element is of the form $g^{\prime}=g h$, with $h \in G_{a_{0}}$. Then the family $s=\hat{s} g$ are the critical sections of $L_{a_{0}}$. Roughly speaking, we locally have the equivalence

$$
\mathcal{E} \mathcal{L}\left(L_{a_{0}}\right)=0 \Longleftrightarrow\left\{\begin{array}{c}
\operatorname{div}^{\mathcal{H}} \frac{\delta l}{\delta \sigma}+\operatorname{ad}_{\sigma \mathcal{H}}^{*} \frac{\delta l}{\delta \sigma}=a \diamond \frac{\delta l}{\delta a}, \\
\nabla^{\sigma} a=0, \\
a(\hat{x})=\{p, \hat{a}\}_{G} \text { with } \hat{a} \in \operatorname{Orb}\left(a_{0}\right), \text { for any } p \in \pi_{M P}^{-1}(\hat{x}), \\
\operatorname{Curv}(\sigma)=0 .
\end{array}\right.
$$

Proof. We have already seen that the conditions on the right hand side of (7.9) are necessary. For the converse, if the holonomy of $\sigma$ is trivial, let $s$ be an integral section. If we write $a(x)=\{s(x), \hat{a}(x)\}_{G}$, we see that $\hat{a}$ is constant from the condition $\nabla^{\sigma} a=0$. With the condition on $\hat{x}$, we have that $\hat{a} \in \operatorname{Orb}\left(a_{0}\right)$. Then, from Theorem 3 , every section $s=\hat{s} g$, with $\hat{a}=g a_{0}$, is a critical section of $L_{a_{0}}$, since $\sigma=\pi_{C J}\left(j^{1} s\right), a=\left\{s(x), a_{0}\right\}_{G}$ and $\lambda=(\sigma, a)$ satisfies (7.8).

\section{Examples}

8.1. Classical mechanics. This is the case when the dimension of the base manifold $M$ is 1 . Let $\pi_{M P}: P=\mathbb{R} \times G \rightarrow \mathbb{R}$ be the trivial principal bundle with structure group $G$ and let $H$ be a subgroup of $G$. We have the following identifications: $J^{1} P=\mathbb{R} \times T G$, $P / H=\mathbb{R} \times(G / H)$, and $\left(J^{1} P\right) / H=\mathbb{R} \times(T(G / H) \oplus \tilde{\mathfrak{h}})$. The last one is a modification of (3.2) with the aid of a connection on the principal bundle $G \rightarrow G / H$ instead of on the bundle $\mathbb{R} \times G \rightarrow \mathbb{R} \times(G / H)$. The space $\tilde{\mathfrak{h}}$ is also understood as the adjoint bundle of this bundle. The configuration bundle of the reduced problem is the composite bundle $\mathbb{R} \times \tilde{\mathfrak{h}} \rightarrow \mathbb{R} \times(G / H) \rightarrow \mathbb{R}$. A section $\sigma$ of this bundle is denoted by $\sigma(t)=(t, \bar{v}(t))$ and the projected section $\varsigma: \mathbb{R} \rightarrow \mathbb{R} \times(G / H)$ by $\varsigma(t)=(t, r(t))$. Then the Lagrange-Poincaré equations read

$$
\begin{gathered}
\mathcal{E} \mathcal{L}_{\bar{v}}(l)=\left\langle\frac{\delta l}{\delta \bar{v}}, i_{\dot{r}} \tilde{\Omega}\right\rangle, \\
\frac{\nabla}{d t} \frac{\delta l}{\delta \bar{v}}+\operatorname{ad}_{\bar{v}}^{*} \frac{\delta l}{\delta \bar{v}}=0,
\end{gathered}
$$

where $\nabla / d t$ is the covariant derivative induced on $\tilde{\mathfrak{h}}^{*}$ by $\mathcal{H}$. These equations can be found in [5] and [15]. For instance, the description of the dynamics of the heavy top, that is, the motion of a rigid body with a fixed point in gravitational field, fits in this context. In this case $G=S O(3)$ and $H=S O(2)$ is the subgroup of rotations with axis parallel to the gravitational direction. This example is also valid for the theory of semidirect product reduction. If we consider the length $a_{0}$ from the fixed point of the heavy top to its center of mass as a variable, the new Lagrangian $L: T S O(3) \times \mathbb{R}^{3} \rightarrow \mathbb{R}$ is $S O(3)$ 
invariant and we can use the equivalence given in (7.9). See [13] for the discussion of this case.

Another example is the dynamics of the rigid body with (three) internal rotors. For this system, $G=S E(3) \times S^{1} \times S^{1} \times S^{1}$ and the subgroup of symmetries of the Lagrangian is $H=S E(3)$. Finally, for the dynamics of the underwater vehicle, we have $G=S E(3)$ and $H=S E(2) \times \mathbb{R}$. For a description of these systems, see for instance [15].

8.2. Harmonic maps. Let $(M, g)$ be a compact oriented Riemannian manifold, and let $(G, h)$ be a Lie group equipped with a right invariant Riemannian metric. We identify the mappings $\phi: M \rightarrow G$ with the global sections of the trivial principal bundle $P=M \times G$. For each $\phi \in C^{\infty}(M, G)$, we may define the energy $E$ on $C^{\infty}(M, G)$ by $E(\phi)=\int_{M} L\left(j^{1} \phi\right) d x$, for the Lagrangian

$$
L\left(j^{1} \phi\right)=\frac{1}{2}\langle T \phi, T \phi\rangle_{g, h},
$$

where $\langle\cdot, \cdot\rangle_{g, h}$ is the induced metric on $T^{*} M \otimes T G$ by $g$ and $h$. The Euler-Lagrange equations for this Lagrangian are given by $\mathcal{E} \mathcal{L}(\phi)=\operatorname{Tr} \nabla \mathrm{d} \phi=0$, where $\nabla$ is the induced Riemannian covariant derivative on $C^{\infty}\left(T^{*} M \otimes T G\right)$ and $\mathrm{Tr}$ is the trace defined by $g$ ( $c f$. [7]). The solutions of these equations are called harmonic mappings. Clearly $L: J^{1} P \rightarrow \mathbb{R}$ is $G$-invariant. If we consider the trivial flat connection $\mathcal{H}$ on $P \rightarrow M$, the reduced Lagrangian $l: T^{*} M \otimes \mathfrak{g} \rightarrow \mathbb{R}$ is

$$
l(\sigma)=\frac{1}{2}\langle\sigma, \sigma\rangle_{g, h},
$$

where $h$ is an inner product on $\mathfrak{g}$. The Euler-Poincaré equation reads in this case

$$
\left\langle\mathrm{d}^{*} \sigma, \cdot\right\rangle_{h}+\operatorname{ad}_{\sigma}^{*}\langle\sigma, \cdot\rangle_{h}=0,
$$

where $\mathrm{d}^{*}=* \mathrm{~d} *$ stands for the codifferential defined by the metric $g$. If the metric $h$ is also left invariant, we have $\operatorname{ad}_{A}^{*}\langle B, \cdot\rangle_{h}+\operatorname{ad}_{B}^{*}\langle A, \cdot\rangle_{h}=0$, for all $A, B \in \mathfrak{g}$, and then the Euler-Poincaré equation is simply

$$
\mathrm{d}^{*} \sigma=0 .
$$

This equation, as well as its corresponding compatibility condition $\operatorname{Curv}(\sigma)=0$ ( $c f$. Theorem 1) are proved for the first time in [23] and are used, for example, in [12] for $G=S U(2)$ and the geometry of $S^{3}$, in [28] for $G=U(n)$, in [18] for $G=S U(n)$ or $S O(n)$, and in [20] for $G=S E(3)$ in applications for robotics mechanisms. The more general formula (8.1) was shown for the first time in [6].

8.3. Harmonic maps: Partial symmetries. We shall use the same notations as in the previous example. Assume now that the metric $h$ is only invariant under the action of a proper subgroup $H$ of $G$. In this case we work with the mechanical connection $\mathcal{H}$ on the principal bundle $\pi_{H G}: G \rightarrow G / H$, which is the connection whose horizontal distribution at every $g \in G$ is the orthogonal complement of the tangent space of the $H$-orbit, that is, $\mathcal{H}_{g}=\left(V_{g}\right)^{\perp}, g \in G$, where $V$ is the vertical distribution. This connection defines a metric $\hat{h}$ on $G / H$ by simply setting $\hat{h}(X, Y):=h\left(X^{\mathcal{H}}, Y^{\mathcal{H}}\right), X, Y \in T_{\zeta}(P / H)$ (see 
Sect. 2.1.3). The harmonic Lagrangian $L: J^{1}(M, G) \rightarrow \mathbb{R}, L\left(j^{1} \phi\right)=\frac{1}{2}\langle T \phi, T \phi\rangle_{g, h}$ projects to the reduced Lagrangian $l: J^{1}(M, G / H) \times T^{*} M \otimes \tilde{\mathfrak{h}} \rightarrow \mathbb{R}$, defined by

$$
l\left(j_{x}^{1} \varsigma, \sigma\right)=\frac{1}{2}\langle T \varsigma, T \varsigma\rangle_{g, \hat{h}}+\frac{1}{2}\langle\sigma, \sigma\rangle_{g, h},
$$

with derivative $\delta l / \delta \sigma=\langle\sigma, \cdot\rangle_{g, h}$. The Lagrange-Poincaré equations and the compatibility condition read

$$
\begin{gathered}
\operatorname{Tr} \nabla \mathrm{d} \varsigma=\left\langle\langle\sigma, \cdot\rangle_{g, h}, i_{\zeta *} \tilde{\Omega}^{\mathcal{H}}\right\rangle, \\
\left\langle\operatorname{div}^{\mathcal{H}} \sigma, \cdot\right\rangle_{h}+\left\langle\sigma, \operatorname{ad}_{\sigma} \cdot\right\rangle_{g}=0, \\
\Omega^{\mathcal{H}}-\nabla^{\mathcal{H}} \sigma+[\sigma, \sigma]=0, \quad \text { along } \pi^{-1}(\varsigma(M)) .
\end{gathered}
$$

In [7, Theorem 4.13] a result for Riemannian submersions (which includes the projections of principal fiber bundles as a special case) studies the relation of the problem defined by the harmonic Lagrangian $L$ and the reduced problem defined by $l$ when $\phi$ is "horizontal", that is, when $\sigma=0$. Equations (8.2) generalize this situation when this condition on $\phi$ is not imposed.

8.4. Sigma models. A closely related topic to harmonic maps is the so called theory of sigma models. These models are important in theoretical physics for different reasons: they have similar properties to Yang-Mills theories, they contain soliton solutions, they are used as approximative models in particle physics, etc. The only sigma models which are known to be integrable are the sigma models taking values into a Lie group or a homogeneous space $(c f$. $[2,18,21])$. We apply now the Lagrange-Poincaré reduction to this last case.

Let $P \rightarrow M$ be a $G$-principal bundle over a Riemannian manifold $(M, g)$ and $\mathcal{A}$ be a connection on $P \rightarrow M$ with connection form $\omega^{\mathcal{A}}: T P \rightarrow \mathfrak{g}$. Let $H$ be a subgroup of $G$. We assume that there exists a subalgebra $\mathfrak{k}$ of $\mathfrak{g}$ endowed with a metric $k$, such that $\mathfrak{g}=\mathfrak{h} \oplus \mathfrak{k}, \operatorname{Ad}_{h} \mathfrak{k} \subset \mathfrak{k}$ and $k \circ A_{h}=k$ for any $h \in H$ (we say that $H$ is metric reductive). We denote by $\operatorname{pr}_{\mathfrak{h}}: \mathfrak{g} \rightarrow \mathfrak{h}$ and $\operatorname{pr}_{\mathfrak{k}}: \mathfrak{g} \rightarrow \mathfrak{k}$ the projections induced by the given decomposition of $\mathfrak{g}$. The composition $\operatorname{pr}_{\mathfrak{h}} \circ \omega^{\mathcal{A}}$ is a connection one-form $\omega^{\mathcal{H}}$ on the fiber bundle $P \rightarrow P / H$. The Lagrangian $L: J^{1} P \rightarrow \mathbb{R}$,

$$
L\left(j_{x}^{1} s\right)=\frac{1}{2}\left\langle\operatorname{pr}_{\mathfrak{k}} \circ \omega^{\mathcal{A}} \circ T s, \operatorname{pr}_{\mathfrak{k}} \circ \omega^{\mathcal{A}} \circ T s\right\rangle_{g, k},
$$

where $\langle,\rangle_{g, k}$ stands for the metric induced by $g$ and $k$ on the space of $\mathfrak{k}$-valued covectors, is clearly $H$ invariant. The metric $k$ induces a metric on $G / H$ which is $H$-invariant. Since the typical fiber of $\pi_{M \Sigma}: P / H \rightarrow M$ is $G / H$, we have a metric for $\pi_{M \Sigma}$-vertical vectors, that will also be denoted by $k$. The reduced Lagrangian takes the form

$$
l\left(j_{x}^{1} \varsigma, \sigma\right)=\frac{1}{2}\left\langle\nabla^{\mathcal{A}} \varsigma, \nabla^{\mathcal{A}} \varsigma\right\rangle_{g, k}
$$

where $\nabla^{\mathcal{A}}$ is the connection induced by $\mathcal{A}$ on $P / H \rightarrow M$, as this bundle is an associated bundle (see Proposition 1). This is the Lagrangian of the sigma model on the bundle $P / H \rightarrow M$, which is precisely the Lagrangian for harmonic maps on this bundle coupled 
with the connection $\mathcal{A}$. We have that $\delta l / \delta \sigma=0$ and therefore the Lagrange-Poincaré equations read

$$
\mathcal{E L}(l)=0 .
$$

We directly write the standard Euler-Lagrange operator instead of the partial one $\mathcal{E} \mathcal{L}_{\varsigma}(l)=0$ as the Lagrangian $l$ only depends on the variable $\varsigma$ and not on $\sigma$, as one can see from Eq. (8.3). The reduced variational problem imposes conditions on $\sigma$ only through the projected section $\zeta$. The solutions of the reduced variational problem are just harmonic sections of $P / H \rightarrow M$ with respect to $\mathcal{A}$. In this case, the reduction procedure can be used backwards. More precisely, for the study of the system (8.4), one can work with the variational problem defined by $L$. A solution of $\mathcal{E} \mathcal{L}(L)=0$ projects onto a solution of $\mathcal{E} \mathcal{L}(l)=0$ and vice-versa, a solution $\varsigma$ of $\mathcal{E} \mathcal{L}(l)=0$ together with an arbitrary section $\sigma$ of $T^{*} M \otimes \tilde{\mathfrak{h}}$ projecting to $\varsigma$ and such that $\operatorname{Curv}(\mathcal{H}-\sigma)=0$ give a solution of $\mathcal{E} \mathcal{L}(L)=0$. This is the so called "injective" or "lifted" formulation of sigma models. This formulation can be found in [2 (where the condition $\operatorname{Curv}(\mathcal{H}-\sigma)=0$ is seen as a conservation law), 21, and 22].

8.5. $K d V$ as a reduced equation. We now give a more concrete example. Let $G=\mathbb{R}^{2}$ with coordinates $(\phi, \psi), M=\mathbb{R}^{2}$ with coordinates $(x, t)$, and $P=M \times G$. The Lagrangian $L: J^{1} P \rightarrow \mathbb{R}, L\left(j_{x}^{1} s\right)=\frac{1}{2} \phi_{t} \phi_{x}+\phi_{x}^{3}+\phi_{x} \psi_{x}+\frac{1}{2} \psi^{2}$ is invariant under the action of the subgroup $H=\mathbb{R}$ acting by translations on the first variable $\phi$.

We consider the trivial connection on the bundle $G=\mathbb{R}^{2} \rightarrow \mathbb{R}=G / H$. In this case we have $P / H=M \times \mathbb{R}, T^{*} M \otimes \tilde{\mathfrak{h}}=T^{*} M \times \mathbb{R}$, with coordinates $(x, t, u, v, \psi)$, and the reduced Lagrangian $l: J^{1}(P / H) \times T^{*} M \otimes \tilde{\mathfrak{h}} \rightarrow \mathbb{R}, l\left(j_{x}^{1} \varsigma, \sigma\right)=\frac{1}{2} v u+u^{3}+u \psi_{x}+\frac{1}{2} \psi^{2}$. Then $\delta l / \delta \sigma=\left(\frac{1}{2} v+3 u^{2}+\psi_{x}\right) \partial / \partial x+\frac{1}{2} u \partial / \partial t$ and the Lagrange-Poincaré equations take the form

$$
\begin{aligned}
\mathcal{E} \mathcal{L}_{\psi}(l) & =\psi-\frac{\partial u}{\partial x}=0, \\
\operatorname{div}\left(\frac{\delta l}{\delta \sigma}\right) & =\frac{1}{2} \frac{\partial v}{\partial x}+6 u \frac{\partial u}{\partial x}+\frac{\partial^{2} \psi}{\partial x^{2}}+\frac{1}{2} \frac{\partial u}{\partial t}=0,
\end{aligned}
$$

with the compatibility condition $\mathrm{d}(u \mathrm{~d} x+v \mathrm{~d} t)=0$, which reads $\partial u / \partial t=\partial v / \partial x$. Rewriting these equations we have

$$
\begin{gathered}
\frac{\partial u}{\partial y}=\frac{\partial v}{\partial x}, \quad \psi=\frac{\partial u}{\partial x}, \\
\frac{\partial u}{\partial t}+6 u \frac{\partial u}{\partial x}+\frac{\partial^{3} u}{\partial x^{3}}=0
\end{gathered}
$$

where one recognizes the $\mathrm{KdV}$ equation in the third equation. In fact, this is the standard way (see for example [19]) to present the $\mathrm{KdV}$ equation by means of a first order Lagrangian, although the use of the symmetry under $\phi$-translations is needed in order to obtain it from $L$, as we have just done. The first group of equations is needed to recover the solutions of the original Lagrangian starting from a solution of the $\mathrm{KdV}$ equation.

Acknowledgements. MCL was partially supported by the DGESIC (Spain) under grant no. BMF20001314. TSR was partially supported by the European Commission and the Swiss Federal Government through funding for the Research Training Network Mechanics and Symmetry in Europe (MASIE) as well as the Swiss National Science Foundation. 


\section{References}

1. Anderson, I.M., Fels, M.E.: Symmetry reduction of variational bicomplexes and the principle of symmetric criticality. Am. J. Math. 119, 609-670 (1997)

2. Bordemann, M., Forger, M., Laartz, J., Schäper, U.: The Lie-Poisson structure of integrable classical non-linear sigma models. Commun. Math. Phys. 152, 167-190 (1993)

3. Castrillón López, M., Munoz Masqué, J.: The geometry of the bundle of connections. Math. Z. 236(4), 797-811 (2001)

4. Castrillón López, M., Ratiu, T.S., Shkoller, S.: Reduction in principal fiber bundles: Covariant EulerPoincaré equations. Proc. Am. Math. Soc. 128(7), 2155-2164 (2000)

5. Cendra, H., Marsden, J., Ratiu, T.: Lagrangian reduction by stages. Mem. Am. Math. Soc. 152(722), (2001)

6. Dai, Y., Shoji, M., Urakawa, H.: Harmonic maps into Lie groups and homogeneous spaces. Diff. Geom. Appl. 7, 143-160 (1997)

7. Eells, J., Ratto, A.: Harmonic maps and minimal immersions with symmetries. Princeton, NJ: Princeton University Press, 1993

8. García Pérez, P.L.: Gauge algebras, curvature and symplectic structure. J. Diff. Geom. 12, 209-227 (1977)

9. Giachetta, G., Mangiarotti, L., Sardanashvily, G.: New lagrangian and hamiltonian methods in field theory. Singapore: World Scientific Co., 1997

10. Goldschmidt, H., Sternberg, S.: The Hamilton-Cartan formalism in the Calculus of Variations. Ann. Inst. Fourier 23, 203-267 (1973)

11. Hamel, G.: Die Lagrange-Eulerschen Gleichungen der Mechanik. Z. Mathematick und Physik 50, 1-57 (1904)

12. Hitchin, N.: Harmonic maps from a 2-torus to the 3-sphere. J. Diff. Geom. 31, 627-710 (1990)

13. Holm, D., Marsden, J., Ratiu, T.: The Euler-Poincaré equations and semidirect products with applications to continuum theories. Adv. in Math. 137, 1-81 (1998)

14. Kobayashi, S., Numizu, K.: Foundations of differential geometry. New York: John Wiley \& Sons, Inc. (Interscience Division), New York, Volume I, 1963; Volume II, 1969

15. Marsden, J.E., Ratiu, T.S.: Introduction to mechanics and symmetry. New York: Springer-Verlag, Inc., 1994

16. Marsden, J.E., Ratiu, T.S., Weinstein, A.: Semi-direct products and reduction in mechanics. Trans. Am. Math. Soc. 281(1), 147-177 (1984)

17. Marsden, J.E., Ratiu, T.S., Weinstein, A.: Reduction and Hamiltonian structures on duals of semidirect product Lie algebras. In Fluids and Plasmas: Geometry and Dynamics, Cont. Math. 28, Providence, RI: Am. Math. Soc. 1984, pp. 55-100

18. Matos, T., Nucamendi, U.: $S U(N)$-and $S O(N)$-invariant chiral fields: One- and two-dimensional subspaces. J. Math. Phys. 40(5), 2500-2513 (1999)

19. Nutku, Y.: Hamiltonian formulation of the KdV equation. J. Math. Phys. 25(6) (1984)

20. Park, F.C., Brockett, R.W.: Kinematic dexterity of robotic mechanism. Int. J. Robotics Res. 13, 1-15 (1994)

21. Percacci, R.: Global definition of nonlinear sigma model and some consequences. J. Math. Phys. 22(9), 1892-1895 (1981)

22. Percacci, R.: Geometry of nonlinear filed theories. Singapore: World Scientific Pub. Co., 1986

23. Pluzhnikov, A.I.: Some properties of harmonic mappings in the case of spheres and Lie groups. Sove. Math. Dokl. 27(1) (1983)

24. Poincaré, H.: Sur une forme nouvelle des équations de la mécanique. C.R. Acad. Sci. 132, 369-371 (1901)

25. Ratiu, T.S.: Euler-Poisson equations on Lie algebras and the $N$-dimensional heavy rigid body. Proc. Nat. Acad. Sci. USA 78, 1327-1328 (1981)

26. Sardanashvily, G., Zakharov, O.: On the geometry of spontaneous symmetry breaking. J. Math. Phys. 33 (1992)

27. Saunders, D.J.: The geometry of jet bundles. Cambridge, UK: Cambridge University Press, 1989

28. Uhlenbeck, K.: Harmonic maps into Lie groups (Classical solutions of the chiral model). J. Diff. Geom. 30, 1-50 (1989) 